ALEA, Lat. Am. J. Probab. Math. Stat. 16, 463-486 (2019)

\title{
Connection probabilities in Poisson random graphs with uniformly bounded edges
}

\author{
Alessandra Faggionato and Hlafo Alfie Mimun \\ Department of Mathematics, University 'La Sapienza' \\ P.le Aldo Moro 2, 00185 \\ Rome, Italy \\ E-mail address: \{ faggiona, mimun \} @mat.uniroma1.it
}

\begin{abstract}
We consider random graphs with uniformly bounded edges on a Poisson point process conditioned to contain the origin. In particular we focus on the random connection model, the Boolean model and the Miller-Abrahams random resistor network with lower-bounded conductances. The latter is relevant for the analysis of conductivity by Mott variable range hopping in strongly disordered systems. By using the method of randomized algorithms developed by DuminilCopin et al. we prove that in the subcritical phase the probability that the origin is connected to some point at distance $n$ decays exponentially in $n$, while in the supercritical phase the probability that the origin is connected to infinity is strictly positive and bounded from below by a term proportional to $\left(\lambda-\lambda_{c}\right), \lambda$ being the density of the Poisson point process and $\lambda_{c}$ being the critical density.
\end{abstract}

\section{Introduction}

We take the homogeneous Poisson point process (PPP) $\xi$ on $\mathbb{R}^{d}, d \geq 2$, with density $\lambda$ conditioned to contain the origin. More precisely, $\xi$ is sampled according to the Palm distribution associated to the homogeneous PPP with density $\lambda$, which is the same as sampling a point configuration $\zeta$ according to the homogeneous PPP with density $\lambda$ and setting $\xi:=\zeta \cup\{0\}$.

We start with two random graphs with vertex set $\xi$ : the random connection model $\mathcal{G}_{\mathrm{RC}}=\left(\xi, \mathcal{E}_{\mathrm{RC}}\right)$ with radial connection function $g$ (see Meester and Roy, 1996) and the Miller-Abrahams random resistor network $\mathcal{G}_{\mathrm{MA}}=\left(\xi, \mathcal{E}_{\mathrm{MA}}\right)$ with lower-bounded conductances (above, $\mathcal{E}_{\mathrm{RC}}$ and $\mathcal{E}_{\mathrm{MA}}$ denote the edge sets).

Received by the editors January 11th, 2018; accepted January 22th, 2019.

2010 Mathematics Subject Classification. 60G55, 82B43, 82D30.

Key words and phrases. Poisson Point Process, Random Connection Model, Boolean Model, Mott Variable Range Hopping, Miller-Abrahams Resistor Network, Connection Probability, Randomized Algorithm.

Research supported by PRIN 20155PAWZB "Large Scale Random Structures". 
The edges in $\mathcal{E}_{\mathrm{RC}}$ are determined as follows. Recall that the connection function $g:(0,+\infty) \rightarrow[0,1]$ is a given measurable function. Given a realization $\xi$, for any unordered pair of sites $x \neq y$ in $\xi$ one declares $\{x, y\}$ to be an edge (i.e. one sets $\left.\{x, y\} \in \mathcal{E}_{\mathrm{RC}}\right)$ with probability $g(|x-y|)$, independently from the other pairs of sites. In what follows, we write $\mathbb{P}_{0, \lambda}^{\mathrm{RC}}$ for the law of the above random connection model (shortly, RC model).

We now move to the Miller-Abrahams random resistor network, explaining first the physical motivations. This random resistor network has been introduced in Miller and Abrahams (1960) as an effective model to study the conductivity via Mott variable range hopping in disordered solids, as doped semiconductors, in the regime of strong Anderson localization and low impurity density. It has been further developed in Ambegaokar et al. (1971) to give a more robust derivation of Mott's law for the low temperature asymptotics of the conductivity (see Faggionato and Mathieu, 2008; Faggionato, 2017; Faggionato et al., 2006; Pollak et al., 2013; Shklovskii and Efros, 1984). Recently developed new materials, as new organic doped seminconductors, enter into this class.

The Miller-Abrahams random resistor network is obtained as follows. Given a realization $\xi$ of a generic simple point process, one samples i.i.d. random variables $\left(E_{x}\right)_{x \in \xi}$, called energy marks, and attaches to any unordered pair of sites $x \neq y$ in $\xi$ a filament of conductance (cf. Ambegaokar et al., 1971; Pollak et al., 2013)

$$
\exp \left\{-\frac{2}{\gamma}|x-y|-\frac{\beta}{2}\left(\left|E_{x}\right|+\left|E_{y}\right|+\left|E_{x}-E_{y}\right|\right)\right\} .
$$

Above $\gamma$ denotes the localization length and $\beta$ the inverse temperature (in what follows we take $\gamma=2$ and $\beta=2$ without loss of generality). Note that the skeleton of the resistor network is the complete graph on $\xi$. In the physical context of inorganic doped semiconductors, the relevant distributions of the energy marks have density function $c|E|^{\alpha} d E$ supported on some interval $[-a, a], c$ being the normalization constant, where $\alpha \geq 0$ and $a>0$. In this case, the physical Mott's law states that the conductivity scales as $\exp \left\{-C \beta^{\frac{\alpha+1}{\alpha+1+d}}\right\}$ for some $\beta$-independent constant $C$. We refer to Faggionato (2017) for a conjectured characterization of the constant $C$.

A key tool (cf. Faggionato and Mathieu, 2008) to rigorously upper bound the conductivity of the Miller-Abrahams resistor network is provided by the control on the size of the clusters formed by edges with high conductance, when these clusters remain finite, hence in a subcritical regime. In particular, we are interested in the subgraph given by the edges $\{x, y\}$ such that

$$
|x-y|+\left|E_{x}\right|+\left|E_{y}\right|+\left|E_{x}-E_{y}\right| \leq \zeta,
$$

for some threshold $\zeta>0$ for which the resulting subgraph does not percolate.

We point out that a lower bound of the conductivity would require (cf. Faggionato et al., 2006) a control on the left-right crossings in the above subgraph when it percolates (we will address this problem in a separate work). To catch the constant $C$ in Mott's law for the Miller-Abrahams resistor network on a Poisson point process, one needs more information on the connection probabilities and on the left-right crossings than what used in Faggionato and Mathieu (2008); Faggionato et al. (2006). For the connection probabilities this additional information will be provided by Theorem 1.4 below. 
As discussed in Faggionato (2017), by the scaling properties of the model, instead of playing with $\zeta$ we can fix the threshold $\zeta$ and vary the Poisson density $\lambda$.

We now give a self-contained mathematical definition of $\mathcal{G}_{\mathrm{MA}}=\left(\xi, \mathcal{E}_{\mathrm{MA}}\right)$. To this aim we fix a probability distribution $\nu$ on $\mathbb{R}$ and a threshold $\zeta>0$. Given a realization $\xi$ of the $\lambda$-homogeneous PPP conditioned to contain the origin, we consider afresh a family of i.i.d. random variables $\left(E_{x}\right)_{x \in \xi}$ with common distribution $\nu$. For any unordered pair of sites $x \neq y$ in $\xi$, we declare $\{x, y\}$ to be an edge (i.e. we set $\left.\{x, y\} \in \mathcal{E}_{\mathrm{MA}}\right)$ if $(1.2)$ is satisfied. In what follows, we write $\mathbb{P}_{0, \lambda}^{\mathrm{MA}}$ for the law of the above random graph, and we will refer to this model simply as the MA model.

We introduce the function $h$ defined as

$$
h(u):=P\left(|E|+\left|E^{\prime}\right|+\left|E-E^{\prime}\right| \leq \zeta-u\right), \quad u \in(0,+\infty),
$$

where $E, E^{\prime}$ are i.i.d. random variables with law $\nu$. In what follows we will use the following fact:

Lemma 1.1. The following properties are equivalent:

(i) The function $h$ is not constantly zero;

(ii) The probability measure $\nu$ satisfies

$$
\nu((-\zeta / 2, \zeta / 2))>0
$$

The proof of Lemma 1.1 is given in Section 2.

To state our main results we fix some notation. We write $S_{n}$ for the boundary of the box $[-n, n]^{d}$, i.e. $S_{n}=\left\{x \in \mathbb{R}^{d}:\|x\|_{\infty}=n\right\}$ and we give the following definition:

Definition 1.2. Given a point $x \in \mathbb{R}^{d}$ and given a graph $G=(V, E)$ in $\mathbb{R}^{d}$, we say that $x$ is connected to $S_{n}$ in the graph $G$, and write $x \leftrightarrow S_{n}$, if $x \in V$ and $x$ is connected in $G$ to some vertex $y \in V$ such that (i) $\|y\|_{\infty} \geq n$ if $\|x\|_{\infty} \leq n$ or (ii) $\|y\|_{\infty} \leq n$ if $\|x\|_{\infty}>n$. We say that a point $x \in \mathbb{R}^{d}$ is connected to infinity in $G$, and write $x \leftrightarrow \infty$, if $x \in V$ and for any $\ell>0$ there exists $y \in V$ with $\|y\|_{\infty} \geq \ell$ such that $x$ and $y$ are connected in $G$.

Both the RC model when $0<\int_{0}^{\infty} r^{d-1} g(r) d r<+\infty$ and the MA model when (1.4) is satisfied exhibit a phase transition at some critical density $\lambda_{c} \in(0, \infty)$ :

$$
\left\{\begin{array}{l}
\lambda<\lambda_{c} \Longrightarrow \mathbb{P}_{0, \lambda}^{R C / M A}(0 \leftrightarrow \infty)=0, \\
\lambda>\lambda_{c} \Longrightarrow \mathbb{P}_{0, \lambda}^{R C / M A}(0 \leftrightarrow \infty)>0 .
\end{array}\right.
$$

Above, and in what follows, we do not stress the dependence of the constants on the dimension $d$, the connection function $g$ (for the RC model), the distribution $\nu$ and the threshold $\zeta$ (for the MA model). The above phase transition (1.5) follows from Meester and Roy (1996, Theorem 6.1) for the RC model and from Proposition 2.2 in Section 2 for the MA model.

Following the recent developments in Duminil-Copin et al. (2019b,a) on percolation theory by means of decision trees (random algorithms) we can improve the knowledge of the above phase transition by providing more detailed information on the behavior of the connection probabilities. To state our main result we need to introduce the concept of good function: 
Definition 1.3. A function $f:(0,+\infty) \rightarrow[0,1]$ is called good if $f$ is positive on a subset of positive Lebesgue measure and if there is a finite family of points $0<r_{1}<r_{2}<\cdots<r_{m-1}<r_{m}$ such that (i) $f(r)=0$ for $r \geq r_{m}$ and (ii) $f$ is uniformly continuous on $\left(r_{i}, r_{i+1}\right)$ for all $i=0, \ldots, m-1$, where $r_{0}:=0$.

We point out that the function $h$ defined in (1.3) is weakly decreasing and satisfies $h(u)=0$ for $u>\zeta$. In particular, due to Lemma 1.1, $h$ is positive on a subset of positive Lebesgue measure if and only if (1.4) is satisfied. Moreover, due to Lemma 1.1 , if $\nu$ has a probability density which is bounded and which is strictly positive on a subset of $(-\zeta / 2, \zeta / 2)$ of positive Lebesgue measure, then the function $h$ is good. In particular, if $\nu$ has density function $c|E|^{\alpha} d E$ supported on some interval $[-a, a]$ (as in the physically relevant cases), then $h$ is good.

Theorem 1.4. Consider the random connection model $\mathcal{G}_{\mathrm{RC}}$ with good radial connection function $\mathrm{g}$. Consider the Miller-Abrahams model $\mathcal{G}_{\mathrm{MA}}$, associated to the distribution $\nu$ and the threshold $\zeta$, and assume that the function $h$ defined in (1.3) is good (cf. Lemma 1.1). In both cases, let the vertex set be given by a Poisson point process with density $\lambda$ conditioned to contain the origin.

Then for both models the following holds:

- (Subcritical phase) For any $\lambda<\lambda_{c}$ there exists $c=c(\lambda)>0$ such that

$$
\mathbb{P}_{0, \lambda}^{\mathrm{RC} / \mathrm{MA}}\left(0 \leftrightarrow S_{n}\right) \leq e^{-c n}, \quad \forall n \in \mathbb{N} .
$$

- (Supercritical phase) There exists $C>0$ such that

$$
\mathbb{P}_{0, \lambda}^{\mathrm{RC} / \mathrm{MA}}(0 \leftrightarrow \infty) \geq C\left(\lambda-\lambda_{c}\right), \quad \forall \lambda>\lambda_{c} .
$$

1.1. Extension to other Poisson models. We point out that the arguments presented in the proof of Theorem 1.4 are robust enough to be applied to other random graphs on the Poisson point process with uniformly bounded edge length. We discuss here the Poisson Boolean model $\mathcal{G}_{B}$ (see Meester and Roy, 1996). Let $\nu \neq \delta_{0}$ be a probability distribution with bounded support in $[0, \infty)$. Given a realization $\xi$ of the PPP with density $\lambda$ conditioned to contain the origin, let $\left(A_{x}\right)_{x \in \xi}$ be i.i.d. random variables with common law $\nu$. The graph $\mathcal{G}_{B}=\left(\xi, \mathcal{E}_{B}\right)$ is then defined by declaring $\{x, y\}$, with $x \neq y$ in $\xi$, to be an edge in $\mathcal{E}_{B}$ if and only if $|x-y| \leq A_{x}+A_{y}$. It is known that the model exhibits a phase transition for some $\lambda_{c} \in(0,+\infty)$ as in $(1.5)$.

The reader can check that the proof of Theorem 1.4 for the MA model can be easily adapted to the Boolean model (the latter is even simpler) if one takes now

$$
h(u):=P\left(u \leq A+A^{\prime}\right), \quad u \in(0,+\infty),
$$

where $A, A^{\prime}$ are i.i.d. with law $\nu$, and if one assumes $h$ to be good.

We collect the above observations in the following theorem:

Theorem 1.5. Consider the Poisson Boolean model $\mathcal{G}_{B}$ with radius law $\nu \neq \delta_{0}$ having bounded support and such that the function $h$ defined in (1.8) is good. Let the vertex set be given by a Poisson point process with density $\lambda$ conditioned to contain the origin. Then the thesis of Theorem 1.4 remains true in this context, where $\lambda_{c}$ is the critical density for the Poisson Boolean model (see Meester and Roy, 1996).

We point out that the above result has been obtained, in part with different techniques, in Ziesche (2018). 


\section{Phase transition in the MA model}

In this section we prove Lemma 1.1 and also show that the phase transition (1.5) takes place in the MA model.

We start with Lemma 1.1:

Proof of Lemma 1.1. Let us show that Items (i) and (ii) are equivalent. Suppose first that (1.4) is violated and let $E, E^{\prime}$ be as in (1.3). Then a.s. we have $|E| \geq \zeta / 2$ and $\left|E^{\prime}\right| \geq \zeta / 2$, thus implying that $P\left(|E|+\left|E^{\prime}\right|+\left|E-E^{\prime}\right| \geq \zeta\right)=1$ and therefore $h(u)=0$ for any $u>0$. Suppose now that (1.4) is satisfied. Then it must be $\nu([0, \zeta / 2))>0$ or $\nu((-\zeta / 2,0])>0$. We analyze the first case, the other is similar. Consider the measure $\nu_{*}$ given by $\nu$ restricted to $[0, \zeta / 2)$. Let $\ell$ be the minimum of the support of $\nu_{*}$. Then for each $\delta>0$ it holds $\nu([\ell, \ell+\delta])>0$. Since $\ell<\zeta / 2$ we can fix $\delta>0$ such that $2 \ell+3 \delta<\zeta$. Take now $E, E^{\prime}$ i.i.d. random variables with law $\nu$. If $E, E^{\prime} \in[\ell, \ell+\delta]$, then $|E|+\left|E^{\prime}\right|+\left|E-E^{\prime}\right| \leq 2 \ell+3 \delta \leq \zeta-u$ for any $u>0$ such that $2 \ell+3 \delta \leq \zeta-u$ (such a $u$ exists). This implies that $h(u) \geq P\left(E, E^{\prime} \in[\ell, \ell+\delta]\right)=\nu([\ell, \ell+\delta])^{2}>0$, hence $h$ is not constantly zero. This completes the proof that Items (i) and (ii) are equivalent.

Remark 2.1. We point out that in the above proof we have shown the following technical fact which will be used in the proof of Proposition 2.2. If $\nu([0, \zeta / 2))>0$, then there are $\ell \geq 0$ and $\delta>0$ such that (i) $2 \ell+3 \delta<\zeta$, (ii) $\nu([\ell, \ell+\delta])>0$, (iii) if $e, e^{\prime} \in[\ell, \ell+\delta]$ then $u+|e|+\left|e^{\prime}\right|+\left|e-e^{\prime}\right| \leq \zeta$ for any $u \in(0, \zeta-2 \ell-3 \delta]$. On the other hand, if $\nu((-\zeta / 2,0])>0$, then there are $\ell \geq 0$ and $\delta>0$ such that (i) $2 \ell+3 \delta<\zeta$, (ii) $\nu([-\ell-\delta,-\ell])>0$, (iii) if $e, e^{\prime} \in[-\ell-\delta,-\ell]$ then $u+|e|+\left|e^{\prime}\right|+\left|e-e^{\prime}\right| \leq \zeta$ for any $u \in(0, \zeta-2 \ell-3 \delta]$. Note that, due to Lemma 1.1, when $h \not \equiv 0$ the above two cases are exhaustive.

Proposition 2.2. There exists $\lambda_{c} \in(0,+\infty)$ such that the phase transition (1.5) takes place in the MA model when $h$ is not constantly zero, equivalently when (1.4) holds (cf. Lemma 1.1).

The proof of the above proposition is a generalization of the one given in Faggionato (2017), in which $\nu$ is the physically relevant distribution $\nu=c|E|^{\alpha} d E$.

Proof: Since two Poisson point processes (possibly conditioned to contain the origin) with density $\lambda<\lambda^{\prime}$ can be coupled in a way that the one with smaller density is contained in the other, we get that the function $\phi(\lambda):=\mathbb{P}_{0, \lambda}^{\mathrm{MA}}(0 \leftrightarrow \infty)$ is weakly increasing. Hence, to get the thesis it is enough to exhibit positive $\lambda_{m}, \lambda_{M}$ such that $\phi\left(\lambda_{m}\right)=0$ and $\phi\left(\lambda_{M}\right)>0$.

Let us consider the graph $\mathcal{G}_{\mathrm{MA}}^{*}=\left(\xi, \mathcal{E}_{\mathrm{MA}}^{*}\right)$ where a pair of sites $x \neq y$ in $\xi$ forms an edge $\{x, y\} \in \mathcal{E}_{\mathrm{MA}}^{*}$ if and only if $|x-y| \leq \zeta$. Trivially, $\mathcal{G}_{\mathrm{MA}} \subset \mathcal{G}_{\mathrm{MA}}^{*}$. On the other hand, by the property of the Poisson Boolean model, the event $\left\{0 \leftrightarrow \infty\right.$ in $\left.\mathcal{G}_{\mathrm{MA}}^{*}\right\}$ has probability zero for $\lambda$ small enough. This proves that $\phi(\lambda)=0$ for $\lambda$ small enough.

Now take $\ell, \delta$ as in Remark 2.1. We treat the case $\nu([0, \zeta / 2))>0$, the complementary case $\nu((-\zeta / 2,0])>0$ is similar. Given a realization $\xi$ of the point process and given random variables $\left(E_{x}\right)_{x \in \xi}$ as in the Introduction, we build a new graph $\hat{\mathcal{G}}_{\mathrm{MA}}=\left(\hat{\mathcal{V}}_{M A}, \hat{\mathcal{E}}_{\mathrm{MA}}\right)$ as follows. As vertex set $\hat{\mathcal{V}}_{M A}$ we take $\left\{x \in \xi: E_{x} \in[\ell, \ell+\delta]\right\}$. We say that a pair of sites $x \neq y$ in $\hat{\mathcal{V}}_{M A}$ forms an edge $\{x, y\} \in \hat{\mathcal{E}}_{\text {MA }}$ if and only if $|x-y| \leq \zeta-2 \ell-3 \delta$. By Remark 2.1 if $\{x, y\} \in \hat{\mathcal{E}}_{\mathrm{MA}}$ then (1.2) is satisfied, and 
therefore $\{x, y\} \in \mathcal{E}_{\mathrm{MA}}$. We have therefore that $\hat{\mathcal{G}}_{\mathrm{MA}} \subset \mathcal{G}_{\mathrm{MA}}$. On the other hand, with positive probability we have $E_{0} \in[\ell, \ell+\delta]$, i.e. $0 \in \hat{\mathcal{V}}_{\mathrm{MA}}$, and conditioning to this event $\hat{\mathcal{G}}_{\mathrm{MA}}$ becomes a Boolean model on a PPP with density $\lambda \nu([\ell, \ell+\delta])$ conditioned to contain the origin, where two points $x, y$ are connected by an edge if and only if $|x-y| \leq \zeta-2 \ell-3 \delta$. By the properties of the Poisson Boolean model (see Meester and Roy, 1996) if $\lambda$ is large enough with positive probability we have $0 \leftrightarrow \infty$ in $\hat{\mathcal{G}}_{\mathrm{MA}}$. Since $\hat{\mathcal{G}}_{\mathrm{MA}} \subset \mathcal{G}_{\mathrm{MA}}$, this proves that $\phi(\lambda)>0$ for $\lambda$ large enough.

\section{Outline of the proof of Theorem 1.4}

In this section we outline the proof of Theorem 1.4. Further details are given in the remaining sections.

Warning 3.1. Without loss of generality we assume, here and in what follows, that $g(r)=0$ for $r \geq 1$ in the RC model, and that $\zeta<1$ in the MA model.

3.1. Probability $\mathbb{P}_{0, \lambda}$ and $\mathbb{P}_{\lambda}$. We write $\mathcal{N}$ for the space of possible realizations of a point process in $\mathbb{R}^{d}$ (see Daley and Vere-Jones, 1988). We denote by $P_{\lambda}$ the law on $\mathcal{N}$ of the $\lambda$-homogeneous Poisson point process and by $P_{0, \lambda}$ the associated Palm distribution. As in Meester and Roy (1996, Sections 1.4, 1.5), given $k \in \mathbb{Z}^{d}$ and $n \in \mathbb{N}$, we define the binary cube of order $n$

$$
\mathcal{K}(n, k):=\prod_{i=1}^{d}\left(k_{i} 2^{-n},\left(k_{i}+1\right) 2^{-n}\right] .
$$

Given $x \in \mathbb{R}^{d}$ there exists a unique binary cube of order $n$, say $\mathcal{K}(n, k(n, x))$, that contains $x$. Moreover, both for $P_{\lambda}$-a.e. $\xi$ and for $P_{0, \lambda}$-a.e. $\xi$, for each $x \in \xi$ there exists a unique smallest number $n(x)$ such that $\mathcal{K}(n(x), k(n(x), x))$ contains no other point of $\xi$.

We then consider a separate probability space $(\Sigma, P)$. For the RC model we take $\Sigma=[0,1]^{\mathcal{R}}, \mathcal{R}=\left\{\left(\left(n_{1}, k_{1}\right),\left(n_{2}, k_{2}\right)\right): n_{1}, n_{2} \in \mathbb{N}, k_{1}, k_{2} \in \mathbb{Z}^{d}\right\}$, and let $P$ be the product probability measure on $\Sigma$ with marginals given by the uniform distribution on $[0,1]$. For the MA resistor network we take $\Sigma=\mathbb{R}^{\mathcal{R}}, \mathcal{R}=\{(n, k),: n \in \mathbb{N}, k \in$ $\mathbb{Z}^{d}$ \}, and let $P$ be the product probability measure on $\Sigma$ with marginals given by $\nu$. Finally, we take the following probabilities on $\mathcal{N} \times \Sigma$ :

$$
\mathbb{P}_{\lambda}:=P_{\lambda} \times P, \quad \mathbb{P}_{0, \lambda}:=P_{0, \lambda} \times P .
$$

We write $\sigma$ for a generic element of $\Sigma$. When treating the RC model, given $x \neq y$ in $\xi$ we shorten the notation by writing $\sigma_{x, y}$ for $\sigma_{\left(n_{1}, k_{1}\right),\left(n_{2}, k_{2}\right)}$ where

$$
\left(n_{1}, k_{1}\right):=(n(x), k(n(x), x)),\left(n_{2}, k_{2}\right):=(n(y), k(n(y), y)) .
$$

Similarly, when treating the MA model, given $x \in \xi$ we write $\sigma_{x}$ for $\sigma_{n, k}$ where $(n, k)=(n(x), k(n(x), x))$.

In what follows we write $\prec_{\text {lex }}$ for the lexicographic order on $\mathbb{R}^{d}$. To a generic element $(\xi, \sigma) \in \mathcal{N} \times \Sigma$ we associate a graph $\mathcal{G}=(\mathcal{V}, \mathcal{E})$ defined as follows. We set $\mathcal{V}:=\xi$ for the vertex set. In the RC model we define the edge set $\mathcal{E}$ as the set of 
pairs $\{x, y\}$ with $x \prec_{\text {lex }} y$ in $\xi$ such that $\sigma_{x, y} \leq g(|x-y|)$. When treating the MA model we define $\mathcal{E}$ as the set of pairs $\{x, y\}$ with $x \neq y$ such that

$$
|x-y|+\left|\sigma_{x}\right|+\left|\sigma_{y}\right|+\left|\sigma_{x}-\sigma_{y}\right| \leq \zeta .
$$

Then the law of $\mathcal{G}(\xi, \sigma)$ with $(\xi, \sigma)$ sampled according to $P_{0, \lambda}$ equals $\mathbb{P}_{0, \lambda}^{\mathrm{RC}}$ in the $\mathrm{RC}$ model, while it equals $\mathbb{P}_{0, \lambda}^{\mathrm{MA}}$ in the MA model. In particular, the phase transition (1.5) can be stated directly for the probability $\mathbb{P}_{0, \lambda}$, and to prove Theorem 1.4 it is enough to consider $\mathbb{P}_{0, \lambda}$ instead of $\mathbb{P}_{0, \lambda}^{R C / M A}$. Note that when $(\xi, \sigma)$ is sampled according to $\mathbb{P}_{\lambda}$, the graph $\mathcal{G}(\xi, \sigma)$ gives a realization of $\mathcal{G}_{\mathrm{RC}} / \mathcal{G}_{\mathrm{MA}}$ with the exception that now $\xi$ is sampled according to a $\lambda$-homogeneous Poisson point process.

3.2. Discretisation. We point out that, due to our assumptions, the graph $\mathcal{G}$ has all edges of length strictly smaller than 1 , both in the $\mathrm{RC}$ model and in the MA model.

Given a positive integer $n$ and given $k=0,1, \ldots, n$, we define the functions

$$
\tilde{\theta}_{k}(\lambda):=\mathbb{P}_{0, \lambda}\left(0 \leftrightarrow S_{k}\right), \quad \tilde{\psi}_{k}(\lambda):=\lambda \tilde{\theta}_{k}(\lambda) .
$$

Warning 3.2. Above, and in what follows, we convey that, when considering $\mathbb{P}_{0, \lambda}$ or the associated expectation $\mathbb{E}_{0, \lambda}$, graphical statements as " $0 \leftrightarrow S_{k}$ " refer to the random graph $\mathcal{G}$, if not stated otherwise. The same holds for $\mathbb{P}_{\lambda}$ and $\mathbb{E}_{\lambda}$.

We have $\tilde{\theta}_{k}(\lambda)=\mathbb{P}_{0, \lambda}\left(0 \leftrightarrow S_{k}\right)=\mathbb{P}_{\lambda}\left(0 \leftrightarrow S_{k}\right.$ in $\left.\mathcal{G}(\xi \cup\{0\}, \sigma)\right)$. Due to Theorem 1.1 in Jiang et al. (2011) (which remains valid when considering the additional random field $\sigma$ ), the derivative $\tilde{\theta}_{n}^{\prime}(\lambda)$ of $\tilde{\theta}_{n}(\lambda)$ can be expressed as follows:

$$
\tilde{\theta}_{n}^{\prime}(\lambda)=\frac{1}{\lambda} \mathbb{E}_{0, \lambda}\left[\left|\operatorname{Piv}_{+}\left(0 \leftrightarrow S_{n}\right) \backslash\{0\}\right|\right],
$$

where $\operatorname{Piv}_{+}\left(0 \leftrightarrow S_{n}\right)$ denotes the set of points which are $(+)$-pivotal for the event $0 \leftrightarrow S_{n}$. We recall that given an event $A$ in terms of the graph $\mathcal{G}$ and a configuration $(\xi, \sigma) \in \mathcal{N} \times \Sigma$, a point $x \in \mathbb{R}^{d}$ is called $(+)$-pivotal for the event $A$ and the configuration $(\xi, \sigma)$, if (i) $x \in \xi$, (ii) the event $A$ takes place for the graph $\mathcal{G}(\xi, \sigma)$, (iii) the event $A$ does not take place in the graph obtained from $\mathcal{G}(\xi, \sigma)$ by removing the vertex $x$ and all edges containing $x$.

Note that $\mathbb{P}_{0, \lambda}\left(0 \in \operatorname{Piv}_{+}\left(0 \leftrightarrow S_{n}\right)\right)=\mathbb{P}_{0, \lambda}\left(0 \leftrightarrow S_{n}\right)=\tilde{\theta}_{n}(\lambda)$. Hence, from $(3.3)$ we get

$$
\tilde{\psi}_{n}^{\prime}(\lambda)=\tilde{\theta}_{n}(\lambda)+\lambda \tilde{\theta}_{n}^{\prime}(\lambda)=\mathbb{E}_{0, \lambda}\left[\left|\operatorname{Piv}_{+}\left(0 \leftrightarrow S_{n}\right)\right|\right] .
$$

The first step in the proof of Theorem 1.4 is to approximate the functions $\tilde{\psi}_{n}(\lambda)$ and $\tilde{\psi}_{n}^{\prime}(\lambda)$ in terms of suitable random graphs built on a grid. To this aim, we introduce the scale parameter $\varepsilon$ of the form $\varepsilon=1 / m$, where $m \geq 2$ is an integer. Moreover we set

$$
\begin{aligned}
& \Lambda_{k}:=[-k, k)^{d}, S_{k}:=\partial \Lambda_{k}=\left\{x \in \mathbb{R}^{d}:\|x\|_{\infty}=k\right\} \\
& R_{x}^{\varepsilon}:=x+[0, \varepsilon)^{d} \text { where } x \in \varepsilon \mathbb{Z}^{d}, \\
& \Gamma_{\varepsilon}:=\left\{x \in \varepsilon \mathbb{Z}^{d} \mid R_{x}^{\varepsilon} \subset \Lambda_{n+1}\right\},
\end{aligned}
$$

and

$$
W_{\varepsilon}:= \begin{cases}\left\{\{x, y\} \mid x \neq y \text { in } \Gamma_{\varepsilon}, g(|x-y|)>0\right\} & \text { for the RC model } \\ \Gamma_{\varepsilon} & \text { for the MA model }\end{cases}
$$


We then consider the product space $\Omega_{\varepsilon}:=\{0,1\}^{\Gamma_{\varepsilon}} \times \mathbb{R}^{W_{\varepsilon}}$ and write $\left(\eta^{\varepsilon}, \sigma^{\varepsilon}\right)$ for a generic element of $\Omega_{\varepsilon}$. We endow $\Omega_{\varepsilon}$ with the product probability measure $\mathbb{P}_{\lambda}^{(\varepsilon)}$ making $\eta_{x}^{\varepsilon}$, as $x$ varies in $\Gamma_{\varepsilon}$, a Bernoulli random variable with parameter

$$
\mathbb{P}_{\lambda}^{(\varepsilon)}\left(\eta_{x}^{\varepsilon}=1\right)=p_{\lambda}(\varepsilon):=\frac{\lambda \varepsilon^{d}}{1+\lambda \varepsilon^{d}}
$$

and making $\sigma_{w}^{\varepsilon}$, as $w$ varies in $W_{\varepsilon}$, a random variable with uniform distribution on $[0,1]$ when considering the $\mathrm{RC}$ model, and with distribution $\nu$ when considering the MA model. To $\left(\eta^{\varepsilon}, \sigma^{\varepsilon}\right) \in \Omega_{\varepsilon}$ we associate the graph $G_{\varepsilon}=\left(V_{\varepsilon}, E_{\varepsilon}\right)$ built as follows. We set

$$
V_{\varepsilon}:=\left\{x \in \Gamma_{\varepsilon}: \eta_{x}^{\varepsilon}=1\right\}
$$

In the $\mathrm{RC}$ model we take

$$
E_{\varepsilon}:=\left\{\{x, y\}: x \neq y \text { in } V_{\varepsilon}, x \prec_{\operatorname{lex}} y, \sigma_{x, y}^{\varepsilon} \leq g(|x-y|)\right\}
$$

and in the MA model we take

$$
E_{\varepsilon}:=\left\{\{x, y\}: x \neq y \text { in } V_{\varepsilon},|x-y|+\left|\sigma_{x}^{\varepsilon}\right|+\left|\sigma_{y}^{\varepsilon}\right|+\left|\sigma_{x}^{\varepsilon}-\sigma_{y}^{\varepsilon}\right| \leq \zeta\right\} .
$$

Given an event $A$ concerning the graph $G_{\varepsilon}$, we define $\operatorname{Piv}(A)$ as the family of sites of $\Gamma_{\varepsilon}$ which are pivotal for the event $A$. More precisely, given a configuration $\left(\eta^{\varepsilon}, \sigma^{\varepsilon}\right)$ in $\Omega_{\varepsilon}$ and a site $x \in \Gamma_{\varepsilon}$, we say that $x$ is pivotal for $A$ if

$$
\mathbb{1}_{A}\left(\eta^{\varepsilon}, \sigma^{\varepsilon}\right) \neq \mathbb{1}_{A}\left(\eta^{\varepsilon, x}, \sigma^{\varepsilon}\right),
$$

where $\eta^{\varepsilon, x}$ is obtained from $\eta^{\varepsilon}$ by replacing $\eta_{x}^{\varepsilon}$ with $1-\eta_{x}^{\varepsilon}$. We point out that the event $\{x \in \operatorname{Piv}(A)\}$ and the random variable $\eta_{x}^{\varepsilon}$ (under $\mathbb{P}_{\lambda}^{(\varepsilon)}$ ) are independent.

In what follows, we write $\mathbb{E}_{\lambda}^{(\varepsilon)}$ for the expectation associated to $\mathbb{P}_{\lambda}^{(\varepsilon)}$ and (recall Definition 1.2) we set

$$
\tilde{\theta}_{k}^{(\varepsilon)}(\lambda):=\mathbb{P}_{\lambda}^{(\varepsilon)}\left(0 \leftrightarrow S_{k} \mid \eta_{0}^{\varepsilon}=1\right), \quad \theta_{k}^{(\varepsilon)}(\lambda):=\mathbb{P}_{\lambda}^{(\varepsilon)}\left(0 \leftrightarrow S_{k}\right) .
$$

Warning 3.3. Above, and in what follows, we convey that, when considering $\mathbb{P}_{\lambda}^{(\varepsilon)}$ or the associated expectation $\mathbb{E}_{\lambda}^{(\varepsilon)}$, graphical statements as " $0 \leftrightarrow S_{k}$ " refer to the random graph $G_{\varepsilon}$, if not stated otherwise.

The following result allows to approximate the functions in (3.2) and their derivatives by their discretized versions:

Proposition 3.4. For any $n \geq 1$ and for all $k=0,1, \ldots, n$ it holds

$$
\begin{aligned}
\tilde{\theta}_{k}(\lambda) & =\lim _{\varepsilon \downarrow 0} \tilde{\theta}_{k}^{(\varepsilon)}(\lambda), \\
\tilde{\psi}_{n}^{\prime}(\lambda) & =\lim _{\varepsilon \downarrow 0} \mathbb{E}_{\lambda}^{(\varepsilon)}\left[\left|\operatorname{Piv}\left(0 \leftrightarrow S_{n}\right)\right|\right] .
\end{aligned}
$$

In particular, it holds $\tilde{\psi}_{k}(\lambda)=\lambda \lim _{\varepsilon \downarrow 0} \tilde{\theta}_{k}^{(\varepsilon)}(\lambda)$.

The last statement in Proposition 3.4 is an immediate consequence of (3.7). The proof of (3.7) is given in Section 6, while the proof of (3.8) is given in Section 7. 
3.3. A crucial inequality on $\theta_{n}^{(\varepsilon)}(\lambda)$. As explained in Duminil-Copin et al. (2019a), due to the phase transition (1.5), to prove Theorem 1.4 it is enough to show that given $\delta \in(0,1)$ there exists a positive constant $c_{0}=c_{0}(\delta)$ such that for each $n \geq 1$

$$
\tilde{\psi}_{n}(\lambda) \leq c_{0} \frac{\sum_{k=0}^{n-1} \tilde{\psi}_{k}(\lambda)}{n} \tilde{\psi}_{n}^{\prime}(\lambda), \quad \forall \lambda \in\left[\delta, \delta^{-1}\right] .
$$

Indeed, since the functions $\lambda \mapsto \tilde{\psi}_{k}(\lambda)$ are increasing in $\lambda$ and converging as $k \rightarrow \infty$, due to Lemma 3 in Duminil-Copin et al. (2019a) applied to the functions $f_{n}(\lambda):=$ $c_{0} \tilde{\psi}_{n}(\lambda),(3.9)$ implies that there exists $\lambda_{*} \in\left[\delta, \delta^{-1}\right]$ fulfilling the following property for any $\lambda \in\left[\delta, \delta^{-1}\right]$ :

$$
\begin{cases}\lambda \tilde{\theta}_{n}(\lambda) \leq M e^{-c n} & \text { if } \lambda<\lambda_{*} \text { and } n \in \mathbb{N}, \\ \lambda \tilde{\theta}(\lambda) \geq C\left(\lambda-\lambda_{*}\right) & \text { if } \lambda>\lambda_{*},\end{cases}
$$

where $M=M(\delta)>0, C=C(\delta)>0, c=c(\lambda, \delta)>0$ and $\tilde{\theta}(\lambda)=\lim _{n \rightarrow \infty} \tilde{\theta}_{n}(\lambda)=$ $\mathbb{P}_{0, \lambda}(0 \leftrightarrow \infty)$. By taking $\delta$ small to have $\lambda_{c} \in\left[\delta, \delta^{-1}\right]$, as a byproduct of (1.5) and (3.10) we get the identity $\lambda_{*}=\lambda_{c}$ and the thesis of Theorem 1.4.

Due to Proposition 3.4, we have (3.9) if we prove that, given $\delta \in(0,1)$, there exists a positive constant $c=c(\delta)$ such that

$$
\tilde{\theta}_{n}^{(\varepsilon)}(\lambda) \leq o(1)+c \frac{\sum_{k=0}^{n-1} \tilde{\theta}_{k}^{(\varepsilon)}(\lambda)}{n} \mathbb{E}_{\lambda}^{(\varepsilon)}\left[\left|\operatorname{Piv}\left(0 \leftrightarrow S_{n}\right)\right|\right]
$$

for any $\lambda \in\left[\delta, \delta^{-1}\right]$ and $n \geq 1$, where the term $o(1)$ goes to zero uniformly in $\lambda \in\left[\delta, \delta^{-1}\right]$ as $\varepsilon \downarrow 0$. Since the event $\left\{0 \leftrightarrow S_{k}\right\}$ implies that $\eta_{0}^{\varepsilon}=1$ and since $p_{\lambda}(\varepsilon)=O\left(\varepsilon^{d}\right)$ uniformly in $\lambda \in\left[\delta, \delta^{-1}\right]$, (3.11) is proved whenever we show the following proposition containing the crucial inequality on $\theta_{n}^{(\varepsilon)}(\lambda)$ :

Proposition 3.5. Given $\delta \in(0,1)$, there exists a positive constant $c=c(\delta)$ such that

$$
\theta_{n}^{(\varepsilon)}(\lambda) \leq o\left(\varepsilon^{d}\right)+c \frac{\sum_{k=0}^{n-1} \theta_{k}^{(\varepsilon)}(\lambda)}{n} \mathbb{E}_{\lambda}^{(\varepsilon)}\left[\left|\operatorname{Piv}\left(0 \leftrightarrow S_{n}\right)\right|\right]
$$

for any $\lambda \in\left[\delta, \delta^{-1}\right]$ and $n \geq 1$, where $o\left(\varepsilon^{d}\right) / \varepsilon^{d}$ goes to zero uniformly in $\lambda \in\left[\delta, \delta^{-1}\right]$ as $\varepsilon \downarrow 0$.

3.4. Proof of Proposition 3.5 by the OSSS inequality. It is possible to derive (3.12) by applying the OSSS inequality for product probability spaces (see Theorem 3.1 in O'Donnell et al., 2005 and Remark 5 in Duminil-Copin et al., 2019a). To recall it and fix the notation in our context, we first introduce the index set $I_{\varepsilon}$ as the disjoint union

$$
I_{\varepsilon}:=\Gamma_{\varepsilon} \sqcup W_{\varepsilon} .
$$

Since in the MA model $W_{\varepsilon}=\Gamma_{\varepsilon}$, given $x \in \Gamma_{\varepsilon}$ we write $\dot{x}$ for the site $x$ thought as element of $W_{\varepsilon}$ inside $I_{\varepsilon}$. More precisely, for the MA model it is convenient to slightly change our notation and set $W_{\varepsilon}:=\left\{\dot{x}: x \in \Gamma_{\varepsilon}\right\}$, thus making $W_{\varepsilon}$ and $\Gamma_{\varepsilon}$ disjoint. We will keep the notation $\sigma_{x}^{\varepsilon}$, instead of $\sigma_{\dot{x}}^{\varepsilon}$, since no confusion arises. To have a uniform notation for random variables, given $i \in I_{\varepsilon}$ we set

$$
\gamma_{i}^{\varepsilon}:= \begin{cases}\eta_{i}^{\varepsilon} & \text { if } i \in \Gamma_{\varepsilon} \\ \sigma_{i}^{\varepsilon} & \text { if } i \in W_{\varepsilon}\end{cases}
$$


By construction, $\gamma^{\varepsilon}=\left(\gamma_{i}^{\varepsilon}: i \in I_{\varepsilon}\right)$ is a family of independent random variables with law $\mathbb{P}_{\lambda}^{(\varepsilon)}$.

We consider an algorithm $T$ to establish if the event $\left\{0 \leftrightarrow S_{n}\right\}$ takes place in $G_{\varepsilon}$, having input the values $\gamma_{i}^{\varepsilon}$ 's. At the beginning the algorithm does not reveal (read) all the values $\gamma_{i}^{\varepsilon}$ 's, but it reveals some of them during the execution. The OSSS inequality (cf. Theorem 3.1 in O'Donnell et al., 2005 and Remark 5 in DuminilCopin et al., 2019a) then reads

$$
\operatorname{Var}_{\varepsilon}\left(\mathbb{1}_{\left\{0 \leftrightarrow S_{n}\right\}}\right) \leq \sum_{i \in I_{\varepsilon}} \delta_{i}^{\varepsilon}(T) \operatorname{Inf}_{i}^{\varepsilon}\left(0 \leftrightarrow S_{n}\right)
$$

where the above variance refers to $\mathbb{P}_{\lambda}^{(\varepsilon)}, \delta_{i}^{\varepsilon}(T)$ and $\operatorname{Inf}_{i}^{\varepsilon}\left(0 \leftrightarrow S_{n}\right)$ are respectively the revealment and the influence of $\gamma_{i}^{\varepsilon}$. More precisely, one sets

$$
\begin{aligned}
& \delta_{i}^{\varepsilon}(T):=\mathbb{P}_{\lambda}^{(\varepsilon)}\left(T \text { reveals the value of } \gamma_{i}^{\varepsilon}\right), \\
& \operatorname{Inf}_{i}^{\varepsilon}\left(0 \leftrightarrow S_{n}\right):=\mathbb{P}_{\lambda}^{(\varepsilon)}\left(\mathbb{1}_{\left\{0 \leftrightarrow S_{n}\right\}}\left(\gamma^{\varepsilon}\right) \neq \mathbb{1}_{\left\{0 \leftrightarrow S_{n}\right\}}\left(\gamma^{\varepsilon, i}\right)\right),
\end{aligned}
$$

where $\gamma^{\varepsilon, i}=\left(\gamma_{j}^{\varepsilon, i}: j \in I_{\varepsilon}\right)$ appearing in the second identity is characterized by the following requirements: (a) $\gamma_{j}^{\varepsilon, i}:=\gamma_{j}^{\varepsilon}$ for all $j \neq i$, (b) $\gamma_{i}^{\varepsilon, i}$ has the same distribution of $\gamma_{i}^{\varepsilon}$, (c) $\gamma_{i}^{\varepsilon, i}$ is independent of the family $\gamma^{\varepsilon}$ (with some abuse, we have kept the notation $\mathbb{P}_{\lambda}^{(\varepsilon)}$ for the joint law).

Since $\operatorname{Var}_{\varepsilon}\left(\mathbb{1}_{\left\{0 \leftrightarrow S_{n}\right\}}\right)=\theta_{n}^{(\varepsilon)}(\lambda)\left(1-\theta_{n}^{(\varepsilon)}(\lambda)\right)$, (3.13) implies for any $\varepsilon_{0}>0$ that

$$
\theta_{n}^{(\varepsilon)}(\lambda) \leq c \sum_{i \in I_{\varepsilon}} \delta_{i}^{\varepsilon}(T) \operatorname{Inf}_{i}^{\varepsilon}\left(0 \leftrightarrow S_{n}\right) \quad \forall \varepsilon<\varepsilon_{0}
$$

where $c:=\sup _{\lambda \in\left[\delta, \delta^{-1}\right]} \sup _{\varepsilon \leq \varepsilon_{0}}\left(1-\theta_{1}^{(\varepsilon)}(\lambda)\right)^{-1}$ (note that $\theta_{n}^{(\varepsilon)}(\lambda) \leq \theta_{1}^{(\varepsilon)}(\lambda)$ for $n \geq 1$ ). As $\theta_{1}^{(\varepsilon)}(\lambda) \leq \mathbb{P}_{\lambda}^{(\varepsilon)}\left(\eta_{0}^{\varepsilon}=1\right) \approx \lambda \varepsilon^{d}$, by taking a suitable $\varepsilon_{0}=\varepsilon_{0}(\delta)$, we get that $c$ is strictly positive and that $c$ depends only on $\delta$.

Similarly to Duminil-Copin et al. (2019a), in order to derive (3.12) from (3.14), for each $k=1, \ldots, n$ we construct an algorithm $T_{k}$ to determine if the event $\{0 \leftrightarrow$ $\left.S_{n}\right\}$ occurs such that the following Lemmas 3.6 and 3.7 are valid:

Lemma 3.6. For any $k \in\{1,2, \ldots, n\}$ given $\delta \in(0,1)$ it holds

$$
\sum_{i \in W_{\varepsilon}} \delta_{i}^{\varepsilon}\left(T_{k}\right) \operatorname{Inf} f_{i}^{\varepsilon}\left(0 \leftrightarrow S_{n}\right)=o\left(\varepsilon^{d}\right),
$$

where $o\left(\varepsilon^{d}\right) / \varepsilon^{d}$ goes to zero uniformly in $\lambda \in\left[\delta, \delta^{-1}\right]$ as $\varepsilon \downarrow 0$.

Lemma 3.7. Given $\delta \in(0,1)$ there exists $c=c(\delta)>0$ such that, for any $\lambda \in$ $\left[\delta, \delta^{-1}\right]$ and any $n \geq 1$, it holds

$$
\frac{1}{n} \sum_{k=1}^{n} \delta_{i}^{\varepsilon}\left(T_{k}\right) \leq c \varepsilon^{-d} \frac{1}{n} \sum_{a=0}^{n-1} \theta_{a}^{(\varepsilon)}(\lambda) \quad \forall i \in \Gamma_{\varepsilon} .
$$

The algorithm $T_{k}$ is described in Section 4, while Lemmas 3.6 and 3.7 are proved in Section 5.

From (3.14), by averaging among $k$, we have

$$
\theta_{n}^{(\varepsilon)}(\lambda) \leq c \sum_{i \in I_{\varepsilon}}\left[\frac{1}{n} \sum_{k=1}^{n} \delta_{i}^{(\varepsilon)}\left(T_{k}\right)\right] \operatorname{Inf}_{i}^{\varepsilon}\left(0 \leftrightarrow S_{n}\right)
$$


for any $\varepsilon \leq \varepsilon_{0}(\delta)$ and for $c=c(\delta)$. By combining (3.17) with Lemmas 3.6 and 3.7 we get

$$
\theta_{n}^{(\varepsilon)}(\lambda) \leq o\left(\varepsilon^{d}\right)+c \varepsilon^{-d} \frac{\sum_{k=0}^{n-1} \theta_{k}^{(\varepsilon)}(\lambda)}{n} \sum_{i \in \Gamma_{\varepsilon}} \operatorname{Inf}_{i}^{\varepsilon}\left(0 \leftrightarrow S_{n}\right)
$$

for any $\varepsilon \leq \varepsilon_{0}(\delta)$ and for $c=c(\delta)$.

Hence the crucial inequality (3.12) in Proposition 3.5 follows from (3.18) and the following lemma:

Lemma 3.8. There exists $c=c(\delta)>0$ such that, for each event $A \subset \Omega_{\varepsilon}$ which is increasing in the random variables $\eta_{i}^{\varepsilon}$ 's, it holds

$$
\operatorname{Inf}_{i}^{\varepsilon}(A) \leq c \varepsilon^{d} \mathbb{P}_{\lambda}^{(\varepsilon)}(i \in \operatorname{Piv}(A)) \quad \forall i \in \Gamma_{\varepsilon}, \forall \lambda \in\left[\delta, \delta^{-1}\right] .
$$

The proof of the above lemma is given in Section 5. This concludes the proof of Proposition 3.5.

\section{The algorithm $T_{k}$}

Fixed $k \in\{1, \ldots, n\}$ we are interested in constructing an algorithm $T_{k}$ that determines if the event $\left\{0 \leftrightarrow S_{n}\right\}$ takes place in $G_{\varepsilon}$. We introduce the sets

$$
\begin{aligned}
& L_{\varepsilon}=\left\{\{x, y\}: x \neq y \text { in } \Gamma_{\varepsilon}, f(|x-y|)>0\right\}, \\
& H_{\varepsilon}^{k}=\left\{\{x, y\} \in L_{\varepsilon}: \overline{x y} \text { intersects } S_{k}\right\},
\end{aligned}
$$

where $f:=g$ in the RC model, $f:=h$ in the MA model and $\overline{x y}$ denotes the segment in $\mathbb{R}^{d}$ with extremes $x, y$. For simplicity, we set $x y:=\{x, y\}$ with the convention that $x \prec_{\text {lex }} y$.

We fix an ordering in $L_{\varepsilon}$ such that the elements of $H_{\varepsilon}^{k}$ precede the elements of $L_{\varepsilon} \backslash H_{\varepsilon}^{k}$. Finally, we introduce the random variables $\left(U_{x, y}^{\varepsilon}: x y \in L_{\varepsilon}\right)$ defined on $\left(\Omega_{\varepsilon}, \mathbb{P}_{\lambda}^{(\varepsilon)}\right)$ as follows:

$$
U_{x, y}^{\varepsilon}:=\left\{\begin{array}{l}
\mathbb{1}\left(\sigma_{x, y}^{\varepsilon} \leq g(|x-y|)\right) \text { in the RC model } \\
\mathbb{1}\left(|x-y|+\left|\sigma_{x}^{\varepsilon}\right|+\left|\sigma_{y}^{\varepsilon}\right|+\left|\sigma_{x}^{\varepsilon}-\sigma_{y}^{\varepsilon}\right| \leq \zeta\right) \text { in the MA model } .
\end{array}\right.
$$

Note that, by definition of the edge set $E_{\varepsilon}$ of the graph $G_{\varepsilon}$, we have that $\{x, y\} \in E_{\varepsilon}$ with $x \prec_{\text {lex }} y$ if and only if $x y \in L_{\varepsilon}$ and $\eta_{x}^{\varepsilon}=\eta_{y}^{\varepsilon}=U_{x, y}^{\varepsilon}=1$.

The algorithm is organised by meta-steps parameterised by the elements of $L_{\varepsilon}$. $t(r)$ will be the number of revealed variables up to the $r^{t h}$ meta-step included. At each meta-step the algorithm will provide two sets $F_{r}, V_{r}: V_{r}$ is roughly the set of vertices connected to some edge in $E_{\varepsilon} \cap H_{\varepsilon}^{k}$ discovered up to the $r^{\text {th }}$ meta-step, while $F_{r}$ is roughly the set of edges connected to some edge in $E_{\varepsilon} \cap H_{\varepsilon}^{k}$ discovered up to the $r^{t h}$ meta-step. We recall that $E_{\varepsilon}$ denotes the set of edges of the graph $G_{\varepsilon}$.

\section{Beginning of the algorithm}

First meta-step. Let $x y$ be the first element of $H_{\varepsilon}^{k}$. Reveal the random variables $\eta_{x}^{\varepsilon}$ and $\eta_{y}^{\varepsilon}$. Set $e_{1}:=x, e_{2}:=y$.

- If $\eta_{x}^{\varepsilon} \eta_{y}^{\varepsilon}=0$, then set $\left(F_{1}, V_{1}\right):=(\emptyset, \emptyset)$ and $t(1)=2$, thus completing the first-meta step in this case. 
- If $\eta_{x}^{\varepsilon} \eta_{y}^{\varepsilon}=1$, then in the RC model reveal the random variable $\sigma_{x, y}^{\varepsilon}$ and set $e_{3}:=x y, t(1):=3$, while in the MA model reveal the random variables $\sigma_{x}^{\varepsilon}, \sigma_{y}^{\varepsilon}$ and set $e_{3}:=\dot{x}, e_{4}:=\dot{y}, t(1):=4$. In both cases set

$$
\left(F_{1}, V_{1}\right):= \begin{cases}(\{x y\},\{x, y\}) & \text { if } U_{x, y}^{\varepsilon}=1, \\ (\emptyset, \emptyset) & \text { otherwise }\end{cases}
$$

thus completing the first meta-step in this case.

* End of the first meta-step *

Generic $r^{t h}$ meta-step for $r \geq 2$. Distinguish two cases. If $r \leq\left|H_{\varepsilon}^{k}\right|$, then let $x y$ be the $r^{t h}$ element of $H_{\varepsilon}^{k}$. If $r>\left|H_{\varepsilon}^{k}\right|$, look for the minimum edge $x y$ in $L_{\varepsilon} \backslash H_{\varepsilon}^{k}$ such that $\{x, y\} \cap V_{r-1} \neq \emptyset$. If such an edge does not exist, then set $R_{\text {end }}:=r-1$ and $T_{\text {end }}:=t(r-1)$, all the generic meta-steps are completed hence move to the final step.

Set $N=0$ ( $N$ will play the role of counter).

- If $\eta_{x}^{\varepsilon}$ has not been revealed yet, do the following: reveal the random variable $\eta_{x}^{\varepsilon}$, increase $N$ by +1 , and set $e_{t(r-1)+N}:=x$.

- If $\eta_{y}^{\varepsilon}$ has not been revealed yet, then reveal the random variable $\eta_{y}^{\varepsilon}$, increase $N$ by +1 and set $e_{t(r-1)+N}:=y$.

- If $\eta_{x}^{\varepsilon} \eta_{y}^{\varepsilon}=0$, then set $\left(F_{r}, V_{r}\right):=\left(F_{r-1}, V_{r-1}\right)$ and $t(r):=t(r-1)+N$, thus completing the $r^{\text {th }}$ meta-step in this case.

- If $\eta_{x}^{\varepsilon} \eta_{y}^{\varepsilon}=1$, then:

$\star$ In the RC model reveal the random variable $\sigma_{x, y}^{\varepsilon}$, increase $N$ by +1 , set $e_{t(r-1)+N}:=x y$

$\star$ In the MA model, if $\sigma_{x}^{\varepsilon}$ has not been revealed yet, then reveal it, increase $N$ by +1 , set $e_{t(r-1)+N}:=\dot{x}$. In addition, if $\sigma_{y}^{\varepsilon}$ has not been revealed yet, then reveal it, increase $N$ by +1 , set $e_{t(r-1)+N}:=\dot{y}$.

In both the above $\star$-cases set $t(r):=t(r-1)+N$,

$$
\left(F_{r}, V_{r}\right):= \begin{cases}\left(F_{r} \cup\{x y\}, V_{r} \cup\{x, y\}\right) & \text { if } U_{x, y}^{\varepsilon}=1, \\ \left(F_{r-1}, V_{r-1}\right) & \text { otherwise, }\end{cases}
$$

thus completing the $r^{t h}$ meta-step.

Final step. If $0 \in V_{R_{\text {end }}}$ and there exists a path from 0 to $V_{R_{\text {end }}} \backslash(-n, n)^{d}$ inside the graph $\left(V_{R_{\text {end }}}, F_{R_{\text {end }}}\right)$ then give as output " $0 \leftrightarrow S_{n}$ ", otherwise give as output "0 \& $S_{n}$ ".

\section{End of the algorithm}

We conclude with some comments on the algorithm.

First, since $L_{\varepsilon}$ is finite, the algorithm always stops. Moreover we note that, when the algorithm has to check if $U_{x, y}^{\varepsilon}=1$, this is possible using only the revealed random variables.

By construction, in the algorithm $T_{k}, V_{R_{\text {end }}}:=\left\{x \in \Gamma_{\varepsilon}: x \leftrightarrow S_{k}\right\}$. Moreover, $F_{R_{\text {end }}}$ is the set of edges belonging to some path in $G_{\varepsilon}$ for which there is an edge $\{x, y\}$ such that the segment $\overline{x y}$ intersects $S_{k}$ (we shortly say that the paths intersect $\left.S_{k}\right)$. If $0 \leftrightarrow S_{n}$ then there must be a path in $G_{\varepsilon}$ from 0 to some point $x$ in $\Gamma_{\varepsilon} \backslash(-n, n)^{d}$, and this path must intersect $S_{k}$. As a consequence, if $0 \leftrightarrow S_{n}$ then 
there exists a path from 0 to $V_{R_{\text {end }}} \backslash(-n, n)^{d}$ inside the graph $\left(V_{R_{\text {end }}}, F_{R_{\text {end }}}\right)$. The other implication is trivially fulfilled, hence the output of the algorithm is correct.

Finally, we point out that the revealed random variables are, in chronological order, the ones associated to the indexes $e_{1}, e_{2}, \ldots, \ldots, e_{T_{\text {end }}}$ (in the cases $e_{i}=x$, $e_{i}=\dot{x}$ and $e_{i}=x y$, the associated random variables are given by $\eta_{x}^{\varepsilon}, \sigma_{x}^{\varepsilon}$ and $\sigma_{x, y}^{\varepsilon}$, respectively).

\section{Proof of Lemmas 3.6, 3.7 and 3.8}

In this section we prove Lemmas 3.6, 3.7 and 3.8 which enter in the proof of Proposition 3.5 as discussed in Section 3.4.

To simplify the notation, given $\alpha \in \mathbb{R}$, we will denote by $O\left(\varepsilon^{\alpha}\right)$ any quantity which can be bounded from above by $C \varepsilon^{\alpha}$, where the constant $C$ can depend on $\delta$ but not on the particular value $\lambda \in\left[\delta, \delta^{-1}\right]$. Similarly, we denote by $o(1)$ any quantity which can be bounded from above by $C f(\varepsilon)$, where $\lim _{\varepsilon \downarrow 0} f(\varepsilon)=0$, and both $f$ and $C$ can depend on $\delta$ but not on the particular value $\lambda \in\left[\delta, \delta^{-1}\right]$. We point out that the above quantities could depend on $n$.

5.1. Proof of Lemma 3.6. We consider first the RC model. Recall that in this case $W_{\varepsilon}=L_{\varepsilon}$ (cf. (3.5)). Let $i=\{x, y\} \in L_{\varepsilon}$ with $x \prec_{\operatorname{lex}} y$. If $\sigma_{x, y}^{\varepsilon}$ is revealed by the algorithm, then it must be $\eta_{x}^{\varepsilon}=\eta_{y}^{\varepsilon}=1$. Hence we have $\delta_{i}^{\varepsilon}\left(T_{k}\right) \leq \mathbb{P}_{\lambda}^{(\varepsilon)}\left(\eta_{x}^{\varepsilon}=\eta_{y}^{\varepsilon}=\right.$ $1)=O\left(\varepsilon^{2 d}\right)$. On the other hand, by definition,

$$
\operatorname{Inf}_{i}^{\varepsilon}\left(0 \leftrightarrow S_{n}\right)=\mathbb{P}_{\lambda}^{(\varepsilon)}\left(\mathbb{1}_{A}\left(\gamma^{\varepsilon}\right) \neq \mathbb{1}_{A}\left(\gamma^{\varepsilon, i}\right)\right) \text { with } A:=\left\{0 \leftrightarrow S_{n}\right\} .
$$

If $\mathbb{1}_{A}\left(\gamma^{\varepsilon}\right) \neq \mathbb{1}_{A}\left(\gamma^{\varepsilon, i}\right)$ then it must be $\eta_{0}^{\varepsilon}=1, \eta_{x}^{\varepsilon}=1, \eta_{y}^{\varepsilon}=1$. As a consequence, we get that $\operatorname{Inf}_{i}^{\varepsilon}\left(0 \leftrightarrow S_{n}\right) \leq \mathbb{P}_{\lambda}^{(\varepsilon)}\left(\eta_{0}^{\varepsilon}=1, \eta_{x}^{\varepsilon}=1, \eta_{y}^{\varepsilon}=1\right)$. The last probability is $O\left(\varepsilon^{2 d}\right)$ if the edge $\{x, y\}$ contains the origin (and there are $O\left(\varepsilon^{-d}\right)$ of such edges in $W_{\varepsilon}$ ), while it is $O\left(\varepsilon^{3 d}\right)$ if the edge $\{x, y\}$ does not contain the origin (and there are $O\left(\varepsilon^{-2 d}\right)$ of such edges in $\left.W_{\varepsilon}\right)$. Using that $\delta_{i}^{\varepsilon}\left(T_{k}\right)=O\left(\varepsilon^{2 d}\right)$, we get (3.15).

We now move to the MA model. Let $i=\dot{x} \in W_{\varepsilon}$. If $\sigma_{x}^{\varepsilon}$ is revealed by the algorithm, then it must be $\eta_{x}^{\varepsilon}=1$. Hence, $\delta_{i}^{\varepsilon}\left(T_{k}\right)=O\left(\varepsilon^{d}\right)$. On the other hand, by (5.1), if $\mathbb{1}_{A}\left(\gamma^{\varepsilon}\right) \neq \mathbb{1}_{A}\left(\gamma^{\varepsilon, i}\right)$ then it must be $\eta_{0}^{\varepsilon}=\eta_{x}^{\varepsilon}=1$. Hence, $\operatorname{Inf}_{i}^{\varepsilon}\left(0 \leftrightarrow S_{n}\right)=$ $O\left(\varepsilon^{d}\right)$ if $x=0$ and $\operatorname{Inf}_{i}^{\varepsilon}\left(0 \leftrightarrow S_{n}\right)=O\left(\varepsilon^{2 d}\right)$ if $x \neq 0$. Since $\left|W_{\varepsilon}\right|=O\left(\varepsilon^{-d}\right)$, we get (3.15), thus concluding the proof of Lemma 3.6.

5.2. Proof of Lemma 3.7. In what follows, constants $c_{*}(d), c(d), \ldots$ are positive constants depending only on the dimension $d$. We also write $i \in H_{\varepsilon}^{k}$ if the site $i$ belongs to some edge in $H_{\varepsilon}^{k}$. Since the edges in $H_{\varepsilon}^{k}$ have length smaller than 1 , if $i \in H_{\varepsilon}^{k}$ then $d_{e}\left(i, S_{k}\right)<1$, where $d_{e}(\cdot, \cdot)$ denotes the Euclidean distance. This implies that $\left|\left\{k \in \mathbb{N}: i \in H_{\varepsilon}^{k}\right\}\right| \leq 2$.

We observe that, when $i \notin H_{\varepsilon}^{k}$,

$$
\left\{\eta_{i}^{\varepsilon} \text { is revealed by } T_{k}\right\} \subset\left\{i \leftrightarrow S_{k}\right\} \cup\left\{\exists j \in \Gamma_{\varepsilon} \backslash\{i\}:|i-j|<1, j \leftrightarrow S_{k}\right\} .
$$

Hence we can bound

$$
\delta_{i}^{\varepsilon}\left(T_{k}\right) \mathbb{1}\left(i \notin H_{\varepsilon}^{k}\right) \leq \sum_{j \in \Gamma_{\varepsilon}:|i-j| \leq 1} \mathbb{P}_{\lambda}^{(\varepsilon)}\left(j \leftrightarrow S_{k}\right) .
$$


By translation invariance we have $\mathbb{P}_{\lambda}^{(\varepsilon)}\left(j \leftrightarrow S_{k}\right) \leq \theta_{d\left(j, S_{k}\right)}^{(\varepsilon)}(\lambda)$, where $d\left(j, S_{k}\right)$ denotes the distance in uniform norm between $j$ and $S_{k}$. Note that $d\left(j, S_{k}\right) \leq n$. Hence we can write

$$
\delta_{i}^{\varepsilon}\left(T_{k}\right) \mathbb{1}\left(i \notin H_{\varepsilon}^{k}\right) \leq \sum_{j \in \Gamma_{\varepsilon}:|i-j| \leq 1} \mathbb{P}_{\lambda}^{(\varepsilon)}\left(j \leftrightarrow S_{k}\right) \leq \sum_{j \in \Gamma_{\varepsilon}:|i-j| \leq 1} \theta_{d\left(j, S_{k}\right)}^{(\varepsilon)}(\lambda) .
$$

If the integer $a \geq 0$ satisfies $a \leq d\left(j, S_{k}\right) \leq a+1$, then we can bound $\theta_{d\left(j, S_{k}\right)}^{(\varepsilon)}(\lambda) \leq$ $\theta_{a}^{(\varepsilon)}(\lambda)$. Hence we can write

$$
\delta_{i}^{\varepsilon}\left(T_{k}\right) \mathbb{1}\left(i \notin H_{\varepsilon}^{k}\right) \leq \sum_{a=0}^{n-1} \theta_{a}^{(\varepsilon)}(\lambda)\left|\left\{j \in \Gamma_{\varepsilon}:|i-j| \leq 1, a \leq d\left(j, S_{k}\right) \leq a+1\right\}\right| .
$$

Let us now consider, for a fixed $a$,

$$
\sum_{k=1}^{n}\left|\left\{j \in \Gamma_{\varepsilon}:|i-j| \leq 1, a \leq d\left(j, S_{k}\right) \leq a+1\right\}\right| .
$$

If $|i-j| \leq 1$ and $a \leq d\left(j, S_{k}\right) \leq a+1$, then it must be $a-c_{*} \leq d\left(i, S_{k}\right) \leq a+c_{*}$, for some constant $c_{*}=c_{*}(d)$. Since $k$ varies among the integers, there are at most $c(d)$ values of $k$ for which $a-c_{*} \leq d\left(i, S_{k}\right) \leq a+c_{*}$. For the other values of $k$ the associated addendum in (5.4) is simply zero. We conclude that the sum in (5.4) is bounded by $c(d) \varepsilon^{-d}$. Therefore, averaging (5.3) among $k$, we get

$$
\frac{1}{n} \sum_{k=1}^{n} \delta_{i}^{\varepsilon}\left(T_{k}\right) \mathbb{1}\left(i \notin H_{\varepsilon}^{k}\right) \leq c(d) \varepsilon^{-d} \frac{1}{n} \sum_{a=0}^{n-1} \theta_{a}^{(\varepsilon)}(\lambda) .
$$

On the other hand, by the observation made at the beginning of the proof, we can bound $\sum_{k=1}^{n} \delta_{i}^{\varepsilon}\left(T_{k}\right) \mathbb{1}\left(i \in H_{\varepsilon}^{k}\right) \leq 2$, while

$$
\varepsilon^{-d} \sum_{a=0}^{n-1} \theta_{a}^{(\varepsilon)}(\lambda) \geq \varepsilon^{-d} \theta_{0}^{(\varepsilon)}(\lambda)=\varepsilon^{-d} \mathbb{P}_{\lambda}^{(\varepsilon)}\left(0 \leftrightarrow S_{0}\right)=\varepsilon^{-d} \mathbb{P}_{\lambda}^{(\varepsilon)}\left(\eta_{0}^{\varepsilon}=1\right)=\frac{\lambda}{1+\lambda \varepsilon^{d}} .
$$

We therefore conclude that

$$
\frac{1}{n} \sum_{k=1}^{n} \delta_{i}^{\varepsilon}\left(T_{k}\right) \mathbb{1}\left(i \in H_{\varepsilon}^{k}\right) \leq c(\delta) \varepsilon^{-d} \frac{1}{n} \sum_{a=0}^{n-1} \theta_{a}^{(\varepsilon)}(\lambda) .
$$

The thesis then follows from (5.5) and (5.7).

5.3. Proof of Lemma 3.8. By symmetry we have

$$
\begin{aligned}
\operatorname{Inf}_{i}^{\varepsilon}(A) & =2 \mathbb{P}_{\lambda}^{(\varepsilon)}\left(\mathbb{1}_{A}\left(\gamma^{\varepsilon}\right) \neq \mathbb{1}_{A}\left(\gamma^{\varepsilon, i}\right), \gamma_{i}^{\varepsilon}=1, \gamma_{i}^{\varepsilon, i}=0\right) \\
& =2 \mathbb{P}_{\lambda}^{(\varepsilon)}\left(\mathbb{1}_{A}\left(\gamma^{\varepsilon}\right) \neq \mathbb{1}_{A}\left(\hat{\gamma}^{\varepsilon}\right), \gamma_{i}^{\varepsilon}=1, \gamma_{i}^{\varepsilon, i}=0\right),
\end{aligned}
$$

where the configuration $\hat{\gamma}^{\varepsilon}$ is obtained from $\gamma^{\varepsilon}$ by changing the value of $\gamma_{i}^{\varepsilon}=\eta_{i}^{\varepsilon}$. The inequality $\mathbb{1}_{A}\left(\gamma^{\varepsilon}\right) \neq \mathbb{1}_{A}\left(\hat{\gamma}^{\varepsilon}\right)$ is equivalent to the fact that $i$ is pivotal for the event $A$ and the configuration $\gamma^{\varepsilon}$. Moreover, this event is independent from $\gamma_{i}^{\varepsilon}$, $\gamma_{i}^{\varepsilon, i}$. Hence (5.8) implies that

$$
\operatorname{Inf}_{i}^{\varepsilon}(A) \leq 2 \mathbb{P}_{\lambda}^{(\varepsilon)}\left(i \in \operatorname{Piv}(A), \eta_{i}^{\varepsilon}=1\right) \leq 2 \mathbb{P}_{\lambda}^{(\varepsilon)}(i \in \operatorname{Piv}(A)) p_{\lambda}(\varepsilon) .
$$

This concludes the proof of Lemma 3.8. 


\section{Proof of (3.7) in Proposition 3.4}

In the proof below, constants $c, c_{1}, c_{2} \ldots$ are understood as positive and $\varepsilon^{-}$ independent and they can change from line to line. To simplify the notation, given $\alpha \in \mathbb{R}$, we will denote by $O\left(\varepsilon^{\alpha}\right)$ any quantity which can be bounded from above by $C \varepsilon^{\alpha}$, where the constant $C$ can depend on $\lambda$. Similarly, we denote by $o(1)$ any quantity which can be bounded from above by $C f(\varepsilon)$, where $\lim _{\varepsilon \downarrow 0} f(\varepsilon)=0$, and both $f$ and $C$ can depend on $\lambda$. All the above quantities can depend also on $n$, which is fixed once and for all.

Recall that $n \geq 1$. To simplify the notation we take $k=n$ (the general case is similar). Recall the notation introduced in Section 3.1. We use the standard convention to identify an element $\xi$ of $\mathcal{N}$ with the atomic measure $\sum_{x \in \xi} \delta_{x}$, which will be denoted again by $\xi$. In particular, given $U \subset \mathbb{R}^{d}, \xi(U)$ equals $|\xi \cap U|$. In addition, given $\xi \in \mathcal{N}$ and $x \in \mathbb{R}^{d}$, we define the translation $\tau_{x} \xi$ as the new set $\xi-x$.

We define the events

$$
\begin{aligned}
& A_{\varepsilon}:=\left\{\xi \in \mathcal{N}: \xi\left(R_{x}^{\varepsilon}\right) \in\{0,1\} \quad \forall x \in \Gamma_{\varepsilon}\right\}, \\
& B_{\varepsilon}:=\left\{\xi \in \mathcal{N}: \xi\left(R_{0}^{\varepsilon}\right)=1\right\} .
\end{aligned}
$$

If $\xi\left(R_{x}^{\varepsilon}\right)=1$, then we define $\bar{x}$ as the unique point of $\xi \cap R_{x}^{\varepsilon}$. On the space $\mathcal{N}$ we define the functions

$$
\varphi_{x}^{\varepsilon}=\mathbb{1}\left(\xi\left(R_{x}^{\varepsilon}\right)=1\right), \quad x \in \Gamma_{\varepsilon} .
$$

Recall Warning 3.2 in Section 3.2.

Lemma 6.1. It holds

$$
\tilde{\theta}_{n}(\lambda)=\mathbb{P}_{0, \lambda}\left(0 \leftrightarrow S_{n}\right)=\lim _{\varepsilon \downarrow 0} \mathbb{P}_{\lambda}\left(\overline{0} \leftrightarrow S_{n} \mid B_{\varepsilon}\right) .
$$

Proof: We use the properties of the Campbell measure and Palm distribution stated in Daley and Vere-Jones (1988, Thm. 12.2.II and Eq. (12.2.4)). We apply Eq. (12.2.4) in Daley and Vere-Jones (1988) with

$$
g(x, \xi):=\mathbb{1}\left(x \in R_{0}^{\varepsilon}\right) \int_{\Sigma} P(d \sigma) \mathbb{1}\left(0 \leftrightarrow S_{n} \text { in } \mathcal{G}(\xi, \sigma)\right)
$$

(see the notation of Section 3.1) and get

$$
\begin{aligned}
\lambda \varepsilon^{d} \mathbb{P}_{0, \lambda}\left(0 \leftrightarrow S_{n}\right) & =\lambda E_{0, \lambda}\left[\int_{\mathbb{R}^{d}} d x g(x, \xi)\right]=E_{\lambda}\left[\int_{\mathbb{R}^{d}} \xi(d x) g\left(x, \tau_{x} \xi\right)\right] \\
& =\mathbb{E}_{\lambda}\left[\int_{R_{0}^{\varepsilon}} \xi(d x) \mathbb{1}\left(x \leftrightarrow S_{n}(x)\right)\right]
\end{aligned}
$$

where $S_{n}(x):=S_{n}+x$. We set $N_{\varepsilon}:=\xi\left(R_{0}^{\varepsilon}\right) . N_{\varepsilon}$ is a Poisson random variable with parameter $\lambda \varepsilon^{d}$. We point out that

$$
\begin{aligned}
& \mathbb{E}_{\lambda}\left[\int_{R_{0}^{\varepsilon}} \xi(d x) \mathbb{1}\left(x \leftrightarrow S_{n}(x)\right) \mathbb{1}\left(N_{\varepsilon} \geq 2\right)\right] \leq \mathbb{E}_{\lambda}\left[N_{\varepsilon} \mathbb{1}\left(N_{\varepsilon} \geq 2\right)\right] \\
& =\mathbb{E}_{\lambda}\left[N_{\varepsilon}\right]-\mathbb{P}_{\lambda}\left(N_{\varepsilon}=1\right)=\lambda \varepsilon^{d}\left(1-e^{-\lambda \varepsilon^{d}}\right)=O\left(\varepsilon^{2 d}\right)
\end{aligned}
$$


Moreover, we can bound

$$
\begin{aligned}
\mathbb{P}_{\lambda}\left(\left\{\overline{0} \leftrightarrow S_{n+\varepsilon}\right\} \cap B_{\varepsilon}\right) & \leq \mathbb{E}_{\lambda}\left[\int_{R_{0}^{\varepsilon}} \xi(d x) \mathbb{1}\left(x \leftrightarrow S_{n}(x)\right) \mathbb{1}\left(N_{\varepsilon}=1\right)\right] \\
& \leq \mathbb{P}_{\lambda}\left(\left\{\overline{0} \leftrightarrow S_{n-\varepsilon}\right\} \cap B_{\varepsilon}\right) .
\end{aligned}
$$

Since $\mathbb{P}_{\lambda}\left(B_{\varepsilon}\right)=\lambda \varepsilon^{d}(1+o(1))$, from $(6.3)$, (6.4) and (6.5) we conclude that

$$
\begin{aligned}
& \mathbb{P}_{0, \lambda}\left(0 \leftrightarrow S_{n}\right) \geq \mathbb{P}_{\lambda}\left(\overline{0} \leftrightarrow S_{n+\varepsilon} \mid B_{\varepsilon}\right)+o(1), \\
& \mathbb{P}_{0, \lambda}\left(0 \leftrightarrow S_{n}\right) \leq \mathbb{P}_{\lambda}\left(\overline{0} \leftrightarrow S_{n-\varepsilon} \mid B_{\varepsilon}\right)+o(1) .
\end{aligned}
$$

On the other hand, $\mathbb{P}_{\lambda}\left(\xi\left(\Lambda_{n+\varepsilon} \backslash \stackrel{\circ}{\Lambda-\varepsilon}_{n-1}\right)=O(\varepsilon)\right.$. Since for $\varepsilon$ small (as we assume from now on) the events $\left\{\xi\left(\Lambda_{n+\varepsilon} \backslash \stackrel{\circ}{\Lambda}_{n-\varepsilon}\right) \geq 1\right\}$ and $B_{\varepsilon}$ are independent, we conclude that $\mathbb{P}_{\lambda}\left(\xi\left(\Lambda_{n+\varepsilon} \backslash \stackrel{\circ}{\Lambda-\varepsilon}_{n}\right) \geq 1 \mid B_{\varepsilon}\right)=O(\varepsilon)$. As a consequence, we have

$$
\begin{aligned}
\mathbb{P}_{\lambda}\left(\overline{0} \leftrightarrow S_{n} \mid B_{\varepsilon}\right) & =\mathbb{P}_{\lambda}\left(\overline{0} \leftrightarrow S_{n} \text { and } \xi\left(\Lambda_{n+\varepsilon} \backslash \stackrel{\circ}{\Lambda}_{n-\varepsilon}\right)=0 \mid B_{\varepsilon}\right)+o(1) \\
& \leq \mathbb{P}_{\lambda}\left(\overline{0} \leftrightarrow S_{n+\varepsilon} \mid B_{\varepsilon}\right)+o(1)
\end{aligned}
$$

and

$$
\begin{aligned}
\mathbb{P}_{\lambda}\left(\overline{0} \leftrightarrow S_{n-\varepsilon} \mid B_{\varepsilon}\right) & =\mathbb{P}_{\lambda}\left(\overline{0} \leftrightarrow S_{n-\varepsilon} \text { and } \xi\left(\Lambda_{n+\varepsilon} \backslash \stackrel{\circ}{\Lambda}_{n-\varepsilon}\right)=0 \mid B_{\varepsilon}\right)+o(1) \\
& \leq \mathbb{P}_{\lambda}\left(\overline{0} \leftrightarrow S_{n} \mid B_{\varepsilon}\right)+o(1) .
\end{aligned}
$$

By combining (6.6) with (6.8), and (6.7) with (6.9), we get

$$
\mathbb{P}_{0, \lambda}\left(0 \leftrightarrow S_{n}\right)=\mathbb{P}_{\lambda}\left(\overline{0} \leftrightarrow S_{n} \mid B_{\varepsilon}\right)+o(1)
$$

which is equivalent to $(6.2)$.

We now enlarge the probability space $(\Sigma, P)$ introduced in Section 3.1 as follows. For the RC model the enlarged probability space is obtained from $(\Sigma, P)$ by adding independent uniform random variables $\sigma_{x, y}$ indexed by the pairs $(x, y)$ with $x \neq y$ and such that $x, y \in \Gamma_{\varepsilon}$ for some $\varepsilon=1 / m, m$ being a positive integer. We take these additional random variables independent from the original random variables $\sigma_{\left(n_{1}, k_{1}\right),\left(n_{2}, k_{2}\right)}$ defined in $(\Sigma, P)$. We point out a slight abuse of notation, since in Section 3.1 we have defined $\sigma_{x, y}$ by means of (3.1) when $x, y \in \xi$. On the other hand, the probability that the realization $\xi$ of a Poisson point process has some vertex in $\cup_{m=1}^{\infty} \Gamma_{1 / m}$ is zero, thus implying that the notation $\sigma_{x, y}$ is not ambiguous with probability 1 . For the MA model the enlarged probability space is obtained from $(\Sigma, P)$ by adding i.i.d. random variables $\sigma_{x}$ with distribution $\nu$, indexed by the points $x$ belonging to some $\Gamma_{\varepsilon}$ as $\varepsilon=1 / m$ and $m$ varies among the positive integers. Again the new random variables are independent from the ones previously defined in $(\Sigma, P)$ and again the notation is not ambiguous with probability 1 . To avoid new symbols, we denote by $(\Sigma, P)$ the enlarged probability space and we keep the definition $\mathbb{P}_{\lambda}:=P_{\lambda} \times P, \mathbb{P}_{0, \lambda}:=P_{0, \lambda} \times P$, where now $P$ refers to the enlarged probability space.

Given points $x \neq y$ and $x^{\prime} \neq y^{\prime}$ we define

$$
\psi_{x, y}^{x^{\prime}, y^{\prime}}:= \begin{cases}\mathbb{1}\left(\sigma_{x, y} \leq g\left(\left|x^{\prime}-y^{\prime}\right|\right)\right) & \text { in the RC model } \\ \mathbb{1}\left(\left|x^{\prime}-y^{\prime}\right|+\left|\sigma_{x}\right|+\left|\sigma_{y}\right|+\left|\sigma_{x}-\sigma_{y}\right| \leq \zeta\right) & \text { in the MA model }\end{cases}
$$


We now introduce a new graph $\mathcal{G}_{\varepsilon}=\left(\mathcal{V}_{\varepsilon}, \mathcal{E}_{\varepsilon}\right)$ which is (as the graph $\mathcal{G}$ introduced in Section 3.1) a function of the pair $(\xi, \sigma) \in \mathcal{N} \times \Sigma$. We set

$$
\mathcal{V}_{\varepsilon}:=\left\{x \in \Gamma_{\varepsilon}: \xi\left(R_{x}^{\varepsilon}\right)=1\right\}
$$

while we define $\mathcal{E}_{\varepsilon}$ as

$$
\mathcal{E}_{\varepsilon}=\left\{\{x, y\} \mid x, y \in \mathcal{V}_{\varepsilon}, x \prec_{\operatorname{lex}} y, \Psi_{x, y}^{x, y}=1\right\} .
$$

When the event $A_{\varepsilon}$ (defined at the beginning of the section) takes place we define a new graph $\mathcal{G}_{\varepsilon}^{\#}=\left(\mathcal{V}_{\varepsilon}, \mathcal{E}_{\varepsilon}^{\#}\right)$ as function of $(\xi, \sigma) \in \mathcal{N} \times \Sigma$ by setting

$$
\mathcal{E}_{\varepsilon}^{\#}=\left\{\{x, y\} \mid x, y \in \mathcal{V}_{\varepsilon}, x \prec_{\operatorname{lex}} y, \Psi_{x, y}^{\bar{x}, \bar{y}}=1\right\} .
$$

Similarly, when the event $A_{\varepsilon}$ takes place, we define a new graph $\mathcal{G}_{\varepsilon}^{*}=\left(\mathcal{V}_{\varepsilon}, \mathcal{E}_{\varepsilon}^{*}\right)$ as function of $(\xi, \sigma) \in \mathcal{N} \times \Sigma$ by setting

$$
\mathcal{E}_{\varepsilon}^{*}=\left\{\{x, y\} \mid x, y \in \mathcal{V}_{\varepsilon}, x \prec_{\text {lex }} y, \Psi_{\bar{x}, \bar{y}}^{\bar{x}, \bar{y}}=1\right\} .
$$

We note that $\mathcal{G}_{\varepsilon}^{*}$ is the graph with vertex set $\mathcal{V}_{\varepsilon}$ and with edges given by the pairs $\{x, y\}$ where $x, y$ vary between the sites in $\mathcal{V}_{\varepsilon}$ with $\{\bar{x}, \bar{y}\} \in \mathcal{E}$. Roughly, $\mathcal{G}_{\varepsilon}^{*}$ is the graph obtained from $\mathcal{G}$ restricted to $\Lambda_{n+1}$ by sliding the vertex at $\bar{x}$ (with $x \in \Gamma_{\varepsilon}$ ) to $x$.

Finally we observe that

$$
\begin{aligned}
\mathbb{P}_{\lambda}\left(A_{\varepsilon}^{c} \cap B_{\varepsilon}\right)=\mathbb{P}_{\lambda}\left(B_{\varepsilon}\right. & \left.\cap\left\{\exists y \in \Gamma_{\varepsilon} \backslash\{0\}: \xi\left(R_{y}^{\varepsilon}\right) \geq 2\right\}\right) \\
& \leq \mathbb{P}_{\lambda}\left(B_{\varepsilon}\right) \sum_{y \in \Gamma_{\varepsilon} \backslash\{0\}} \mathbb{P}_{\lambda}\left(\xi\left(R_{y}^{\varepsilon}\right) \geq 2\right)=\mathbb{P}_{\lambda}\left(B_{\varepsilon}\right) O\left(\varepsilon^{d}\right),
\end{aligned}
$$

thus implying that $\mathbb{P}_{\lambda}\left(A_{\varepsilon}^{c} \mid B_{\varepsilon}\right)=O\left(\varepsilon^{d}\right)$.

Lemma 6.2. The event $\left\{\overline{0} \leftrightarrow S_{n}\right.$ in $\left.\mathcal{G}\right\}$ equals the event $\left\{0 \leftrightarrow S_{n}\right.$ in $\left.\mathcal{G}_{\varepsilon}^{*}\right\}$ if $\xi\left(\Lambda_{n+\varepsilon}\right)$ $\stackrel{\circ}{n-\varepsilon}_{n}=0$.

Proof: Let $\{a, b\}$ be an edge of $\mathcal{G}$ with $\|a\|_{\infty}<n$ and $\|b\|_{\infty} \geq n$. Since $\xi\left(\Lambda_{n+\varepsilon} \backslash\right.$ $\left.\stackrel{\circ}{\Lambda-\varepsilon}_{n}\right)=0$ we have $\|a\|_{\infty}<n-\varepsilon$ and $\|b\|_{\infty} \geq n+\varepsilon$. On the other hand, the Euclidean distance between $a$ and $b$ is smaller than 1, thus implying that $\|a\|_{\infty} \geq$ $n+\varepsilon-1$ and $\|b\|_{\infty}<n+1-\varepsilon$. Suppose now that $\overline{0} \leftrightarrow S_{n}$ in $\mathcal{G}$ and let $a, b \in \xi$ be such that $\overline{0} \leftrightarrow a,\{a, b\}$ is an edge of $\mathcal{G},\|a\|_{\infty}<n$ and $\|b\|_{\infty} \geq n$. As already observed, $n+\varepsilon \leq\|b\|_{\infty}<n+1-\varepsilon$, thus implying that $b=\bar{z}$ for some $z \in \Gamma_{\varepsilon}$ and that $n \leq\|z\|_{\infty}<n+1$. Since $0 \leftrightarrow z$ in $\mathcal{G}_{\varepsilon}^{*}$, we conclude that $0 \leftrightarrow S_{n}$ in $\mathcal{G}_{\varepsilon}^{*}$.

Suppose now that $0 \leftrightarrow S_{n}$ in $\mathcal{G}_{\varepsilon}^{*}$. Then there exists $z \in \Gamma_{\varepsilon}$ such that $\|z\|_{\infty} \geq n$, $0 \leftrightarrow z$ in $\mathcal{G}_{\varepsilon}^{*}$. As a consequence, $\overline{0} \leftrightarrow \bar{z}$ in $\mathcal{G}$. Since $\xi\left(\Lambda_{n+\varepsilon} \backslash \stackrel{\circ}{\Lambda}_{n-\varepsilon}\right)=0$, it must be $\bar{z} \in \bar{\Lambda}_{n+\varepsilon}^{c}$, thus implying that $\overline{0} \leftrightarrow S_{n}$ in $\mathcal{G}$.

\section{Lemma 6.3. It holds}

$$
\mathbb{P}_{\lambda}\left(\overline{0} \leftrightarrow S_{n} \mid B_{\varepsilon}\right)=\mathbb{P}_{\lambda}\left(0 \leftrightarrow S_{n} \text { in } \mathcal{G}_{\varepsilon} \mid A_{\varepsilon} \cap B_{\varepsilon}\right)+o(1) .
$$

Proof: Since $\mathbb{P}_{\lambda}\left(A_{\varepsilon}^{c} \mid B_{\varepsilon}\right)=O\left(\varepsilon^{d}\right)$ we can write

$$
\mathbb{P}_{\lambda}\left(\overline{0} \leftrightarrow S_{n} \mid B_{\varepsilon}\right)=\mathbb{P}_{\lambda}\left(\overline{0} \leftrightarrow S_{n} \mid A_{\varepsilon} \cap B_{\varepsilon}\right)+O\left(\varepsilon^{d}\right) .
$$


From now on we suppose the event $A_{\varepsilon} \cap B_{\varepsilon}$ to take place. We want to apply Lemma 6.2. By independence, $\mathbb{P}_{\lambda}\left(\left\{\xi\left(\Lambda_{n+\varepsilon} \backslash \stackrel{\circ}{\Lambda}_{n-\varepsilon}\right) \geq 1\right\} \cap B_{\varepsilon}\right)=O\left(\varepsilon^{d+1}\right)$, while $\mathbb{P}_{\lambda}\left(A_{\varepsilon} \cap B_{\varepsilon}\right) \geq C \varepsilon^{d}$ by (6.14). As a consequence,

$$
\mathbb{P}_{\lambda}\left(\xi\left(\Lambda_{n+\varepsilon} \backslash \stackrel{\circ}{\Lambda}_{n-\varepsilon}\right)=0 \mid A_{\varepsilon} \cap B_{\varepsilon}\right)=1+o(1) .
$$

By the above observation and Lemma 6.2, in the r.h.s. of (6.16) we can replace the event $\left\{\overline{0} \leftrightarrow S_{n}\right.$ in $\left.\mathcal{G}\right\}$ with the event $\left\{0 \leftrightarrow S_{n}\right.$ in $\left.\mathcal{G}_{\varepsilon}^{*}\right\}$ with an error $o(1)$. In particular to get (6.15) it is enough to show that

$$
\mathbb{P}_{\lambda}\left(0 \leftrightarrow S_{n} \text { in } \mathcal{G}_{\varepsilon} \mid A_{\varepsilon} \cap B_{\varepsilon}\right)=\mathbb{P}_{\lambda}\left(0 \leftrightarrow S_{n} \text { in } \mathcal{G}_{\varepsilon}^{*} \mid A_{\varepsilon} \cap B_{\varepsilon}\right)+o(1) .
$$

Since the events $A_{\varepsilon}, B_{\varepsilon}$ do not depend on $\sigma$, and since the random variables of $\sigma$-type are i.i.d. w.r.t. $\mathbb{P}_{\lambda}$ conditioned to $\xi$, we conclude that $\mathcal{G}_{\varepsilon}^{*}$ and $\mathcal{G}_{\varepsilon}^{\#}$ have the same law under $\mathbb{P}_{\lambda}\left(\cdot \mid A_{\varepsilon} \cap B_{\varepsilon}\right)$. Hence, in order to prove (6.17) it is enough to show that

$$
\mathbb{P}_{\lambda}\left(0 \leftrightarrow S_{n} \text { in } \mathcal{G}_{\varepsilon} \mid A_{\varepsilon} \cap B_{\varepsilon}\right)=\mathbb{P}_{\lambda}\left(0 \leftrightarrow S_{n} \text { in } \mathcal{G}_{\varepsilon}^{\#} \mid A_{\varepsilon} \cap B_{\varepsilon}\right)+o(1) .
$$

Trivially, (6.18) follows from Lemma 6.4 below. The result stated in Lemma 6.4 is stronger than what we need here (we do not need the term $\xi\left(\Lambda_{n+2}\right)$ in the expectation), and it is suited for a further application in the next section.

Lemma 6.4. It holds $\mathbb{E}_{\lambda}\left[\xi\left(\Lambda_{n+2}\right) \mathbb{1}\left(\mathcal{G}_{\varepsilon}^{\#} \neq \mathcal{G}_{\varepsilon}\right) \mid A_{\varepsilon} \cap B_{\varepsilon}\right]=o(1)$.

Proof: Recall definition (6.1). Since the graphs $\mathcal{G}_{\varepsilon}$ and $\mathcal{G}_{\varepsilon}^{\#}$ have the same vertex set $\mathcal{V}_{\varepsilon}$, by an union bound we can estimate

$$
\begin{aligned}
& \mathbb{E}_{\lambda}\left[\xi\left(\Lambda_{n+2}\right) \mathbb{1}\left(\mathcal{G}_{\varepsilon}^{\#} \neq \mathcal{G}_{\varepsilon}\right) \mid A_{\varepsilon} \cap B_{\varepsilon}\right] \\
& \leq \sum_{x \prec \operatorname{lex} y \text { in } \Gamma_{\varepsilon}} \mathbb{E}_{\lambda}\left[\xi\left(\Lambda_{n+2}\right) \mathbb{1}\left(\varphi_{x}^{\varepsilon}=1, \varphi_{y}^{\varepsilon}=1, \varphi_{0}^{\varepsilon}=1, \Psi_{x, y}^{\bar{x}, \bar{y}} \neq \Psi_{x, y}^{x, y}\right) \mid A_{\varepsilon} \cap B_{\varepsilon}\right] .
\end{aligned}
$$

Note that $\xi\left(\Lambda_{n+2}\right) \leq \xi\left(\Lambda_{n+2} \backslash\left(R_{x}^{\varepsilon} \cup R_{y}^{\varepsilon} \cup R_{0}^{\varepsilon}\right)\right)+3=: Z$ whenever $\varphi_{x}^{\varepsilon}=\varphi_{y}^{\varepsilon}=\varphi_{0}^{\varepsilon}=$ 1. We also observe that, under $\mathbb{P}_{\lambda}\left(\cdot \mid A_{\varepsilon} \cap B_{\varepsilon}\right)$, the random variables $Z, \varphi_{x}^{\varepsilon}, \varphi_{y}^{\varepsilon}$, $\mathbb{1}\left(\Psi_{x, y}^{\bar{x}, \bar{y}} \neq \Psi_{x, y}^{x, y}\right)$ are independent. As a consequence, (6.19) implies that

$$
\mathbb{E}_{\lambda}\left[\xi\left(\Lambda_{n+2}\right) \mathbb{1}\left(\mathcal{G}_{\varepsilon}^{\#} \neq \mathcal{G}_{\varepsilon}\right) \mid A_{\varepsilon} \cap B_{\varepsilon}\right] \leq c \sum_{x \in \Gamma_{\varepsilon}} \sum_{y \in \Gamma_{\varepsilon} \backslash\{0, x\}} p_{\lambda}(\varepsilon)^{2-\delta_{0, x}} P\left(\Psi_{x, y}^{\bar{x}, \bar{y}} \neq \Psi_{x, y}^{x, y}\right),
$$

where $\delta_{0, x}$ denotes the Kronecker delta.

Above we have used the definition of $p_{\lambda}(\varepsilon)$ given in (3.6), the fact that

$$
\mathbb{P}_{\lambda}\left(\varphi_{z}^{\varepsilon}=1 \mid A_{\varepsilon} \cap B_{\varepsilon}\right)= \begin{cases}1 & \text { if } z=0, \\ p_{\lambda}(\varepsilon) & \text { if } z \in \Gamma_{\varepsilon} \backslash\{0\},\end{cases}
$$

and the estimate (recall that $\left.\mathbb{P}_{\lambda}\left(A_{\varepsilon}^{c} \mid B_{\varepsilon}\right)=o(1)\right)$

$$
\mathbb{E}_{\lambda}\left[Z \mid A_{\varepsilon} \cap B_{\varepsilon}\right] \leq \frac{\mathbb{E}_{\lambda}\left[Z \mathbb{1}\left(B_{\varepsilon}\right)\right]}{\mathbb{P}_{\lambda}\left(A_{\varepsilon} \cap B_{\varepsilon}\right)}=\mathbb{E}_{\lambda}[Z] \frac{\mathbb{P}_{\lambda}\left(B_{\varepsilon}\right)}{\mathbb{P}_{\lambda}\left(A_{\varepsilon} \cap B_{\varepsilon}\right)}=\frac{\mathbb{E}_{\lambda}[Z]}{\mathbb{P}_{\lambda}\left(A_{\varepsilon} \mid B_{\varepsilon}\right)}=O(1) .
$$

From now on we distinguish between the RC model and the MA model. 
- We consider the RC model. Recall that the connection function $g$ is good. To simplify the notation we restrict to $m=2$ and $r_{2}=1$ in Definition 1.3 (the general case is similar). For $i=1,2$, we set

$$
\omega_{i}(\delta):=\sup \left\{|g(a)-g(b)|: a, b \in\left(r_{i-1}, r_{i}\right) \text { and }|a-b| \leq \delta\right\} .
$$

Since $g$ is uniformly continuous in $\left(r_{i-1}, r_{i}\right)$ we know that $\omega_{i}(\delta) \rightarrow 0$ when $\delta \rightarrow 0$, for $i=1,2$. Since $g$ has support inside $(0,1), P\left(\Psi_{x, y}^{\bar{x}, \bar{y}} \neq \Psi_{x, y}^{x, y}\right)=0$ if $|x-y| \geq 1$ and $|\bar{x}-\bar{y}| \geq 1$. By taking $\varepsilon$ small, this always happens if $|x-y| \geq 2$. Hence, in the sum inside (6.19) we can restrict to $x, y$ with $|x-y|<2$.

As a consequence we have

$$
\text { r.h.s. of }(6.20) \leq c \sum_{x \in \Gamma_{\varepsilon}} \sum_{\substack{y \in \Gamma_{\varepsilon} \backslash\{0, x\}: \\|x-y|<2}} p_{\lambda}(\varepsilon)^{2-\delta_{0, x}}|g(|x-y|)-g(|\bar{x}-\bar{y}|)| \text {. }
$$

It remains to prove that the r.h.s. of (6.22) is $o(1)$.

Since $|z-\bar{z}|<\sqrt{d} \varepsilon$ for all $z \in \Gamma_{\varepsilon},|x-y|$ differs from $|\bar{x}-\bar{y}|$ by at most $2 \sqrt{d} \varepsilon$. We set $M_{x, y}:=\max \{|x-y|,|\bar{x}-\bar{y}|\}$ and $m_{x, y}:=\min \{|x-y|,|\bar{x}-\bar{y}|\}$. Note that $m_{x, y}>0$. Since $g$ has support inside $(0,1)$, in $(6.22)$ we can restrict to the case $m_{x, y}<r_{2}=1$. Moreover, if we consider the following cases:

(i) $0=r_{0}<m_{x, y}<M_{x, y}<r_{1}$,

(ii) $r_{1}<m_{x, y}<M_{x, y}<r_{2}=1$,

we can bound

$$
\begin{aligned}
& |g(|x-y|)-g(|\bar{x}-\bar{y}|)| \leq \omega_{1}(2 \sqrt{d} \varepsilon) \text { in the case (i), } \\
& |g(|x-y|)-g(|\bar{x}-\bar{y}|)| \leq \omega_{2}(2 \sqrt{d} \varepsilon) \text { in the case (ii). }
\end{aligned}
$$

As a consequence the contribution in the r.h.s. of (6.22) of the pairs $x, y$ with $x \neq 0$ and $M_{x, y}, m_{x, y}$ which satisfy case (i) or (ii), is bounded by $c \varepsilon^{-2 d} p_{\lambda}(\varepsilon)^{2}\left(\omega_{1}(2 \sqrt{d} \varepsilon)+\right.$ $\left.\omega_{2}(2 \sqrt{d} \varepsilon)\right)=O\left(\omega_{1}(2 \sqrt{d} \varepsilon)+\omega_{2}(2 \sqrt{d} \varepsilon)\right)=o(1)$. Similarly the contribution in the r.h.s. of (6.22) of the pairs $x=0, y$ with $M_{x, y}, m_{x, y}$ which satisfy case (i) or (ii), is $O\left(\omega_{1}(2 \sqrt{d} \varepsilon)+\omega_{2}(2 \sqrt{d} \varepsilon)\right)=o(1)$.

The other pairs $x, y$ in (6.22) we have not considered yet satisfy (a) $m_{x, y} \leq r_{1} \leq$ $M_{x, y}$ or (b) $m_{x, y} \leq r_{2} \leq M_{x, y}$. Defining $r:=r_{1}$ in case (a) and $r:=r_{2}$ in case (b), we can restrict to study the contribution in the r.h.s. of (6.22) of the pairs $x, y$ which satisfy $m_{x, y} \leq r \leq M_{x, y}$. We now estimate such contribution. Since $m_{x, y} \geq|x-y|-2 \sqrt{d} \varepsilon$ and $M_{x, y} \leq|x-y|+2 \sqrt{d} \varepsilon$, it must be

$$
r-2 \sqrt{d} \varepsilon \leq|x-y| \leq r+2 \sqrt{d} \varepsilon .
$$

The number of points $y \in \Gamma_{\varepsilon}$ satisfying (6.23) are of order $O\left(\varepsilon^{-d+1}\right)$, hence the pairs $x, y$ with $m_{x, y} \leq r \leq M_{x, y}$ and $x \neq 0$ are of order $O\left(\varepsilon^{-2 d+1}\right)$ while the pairs $x, y$ with $m_{x, y} \leq r \leq M_{x, y}$ and $x=0$ are $O\left(\varepsilon^{-d+1}\right)$. Bounding in both cases $|g(|x-y|)-g(|\bar{x}-\bar{y}|)|$ by 1 , we conclude that the contribution in the r.h.s. of (6.22) of the pairs $x, y$ with $m_{x, y} \leq r \leq M_{x, y}$ is bounded by $O\left(\varepsilon^{-2 d+1}\right) p_{\lambda}(\varepsilon)^{2}+$ $O\left(\varepsilon^{-d+1}\right) p_{\lambda}(\varepsilon)=o(1)$.

- We consider the MA model. As for (6.22) we have

$$
\text { r.h.s. }(6.20) \leq \sum_{x \in \Gamma_{\varepsilon}} \sum_{\substack{y \in \Gamma_{\varepsilon} \backslash\{0\},|x-y|<2}} p_{\lambda}(\varepsilon)^{2-\delta_{0, x}}|h(|x-y|)-h(|\bar{x}-\bar{y}|)| .
$$


Since by assumption $h$ is good, one can proceed exactly as done for the RC model and conclude that the r.h.s. of (6.24) is of order $o(1)$.

6.1. Conclusion of the proof of (3.7) in Proposition 3.4. By combining Lemmas 6.1 and 6.3 we get that $\tilde{\theta}_{n}(\lambda)=\mathbb{P}_{\lambda}\left(0 \leftrightarrow S_{n}\right.$ in $\left.\mathcal{G}_{\varepsilon} \mid A_{\varepsilon} \cap B_{\varepsilon}\right)+o(1)$. On the other hand, by construction the random graph $\mathcal{G}_{\varepsilon}$ sampled according to $\mathbb{P}_{\lambda}\left(\cdot \mid A_{\varepsilon} \cap B_{\varepsilon}\right)$ has the

same law of the random graph $G_{\varepsilon}$ sampled according to $\mathbb{P}_{\lambda}^{(\varepsilon)}\left(\cdot \mid \eta_{0}^{\varepsilon}=1\right)$. This implies that

$$
\mathbb{P}_{\lambda}\left(0 \leftrightarrow S_{n} \text { in } \mathcal{G}_{\varepsilon} \mid A_{\varepsilon} \cap B_{\varepsilon}\right)=\mathbb{P}_{\lambda}^{(\varepsilon)}\left(0 \leftrightarrow S_{n} \mid \eta_{0}^{\varepsilon}=1\right)=\tilde{\theta}_{n}^{(\varepsilon)}(\lambda)
$$

This completes the proof of (3.7) for $n=k$. As stated at the beginning, the choice $n=k$ was to simplify the notation, the proof is the same for general $k$.

\section{Proof of (3.8) in Proposition 3.4}

We use the same convention on constants $c, c_{1}, c_{2} \ldots$, on $O\left(\varepsilon^{\alpha}\right)$ and $o(1)$ as stated at the beginning of the previous section. Below we restrict to $n \geq 1$.

Due to (3.4) we need to prove that

$$
\mathbb{E}_{0, \lambda}\left[\left|\operatorname{Piv}_{+}\left(0 \leftrightarrow S_{n}\right)\right|\right]=\lim _{\varepsilon \downarrow 0} \mathbb{E}_{\lambda}^{(\varepsilon)}\left[\left|\operatorname{Piv}\left(0 \leftrightarrow S_{n}\right)\right|\right]
$$

(recall the definition of $\operatorname{Piv}_{+}\left(0 \leftrightarrow S_{n}\right)$ given after (3.3)). We define the function $g: \mathbb{R}^{d} \times \mathcal{N} \rightarrow \mathbb{R}$ as

$$
g(x, \xi)=\mathbb{1}\left(x \in R_{0}^{\varepsilon}\right) \int_{\Sigma} P(d \sigma)\left|\operatorname{Piv}_{+}\left(0 \leftrightarrow S_{n}\right)(\xi, \sigma)\right| .
$$

Then, by the property of the Palm distribution and of $P$ (cf. Section 3.1 and Thm. 12.2.II and Eq. (12.2.4) in Daley and Vere-Jones, 1988),

$$
\begin{aligned}
\lambda \varepsilon^{d} \mathbb{E}_{0, \lambda}\left[\left|\operatorname{Piv}_{+}\left(0 \leftrightarrow S_{n}\right)\right|\right] & =\lambda E_{0, \lambda}\left[\int_{\mathbb{R}^{d}} d x g(x, \xi)\right]=\mathbb{E}_{\lambda}\left[\int_{\mathbb{R}^{d}} \xi(d x) g\left(x, \tau_{x} \xi\right)\right]= \\
& =\mathbb{E}_{\lambda}\left[\int_{R_{0}^{\varepsilon}} \xi(d x)\left|\operatorname{Piv}_{+}\left(x \leftrightarrow S_{n}(x)\right)\right|\right] .
\end{aligned}
$$

We recall that $S_{n}(x)=S_{n}+x$. We can write the last member of $(7.2)$ as $\mathcal{C}_{1}+\mathcal{C}_{2}$, with $\mathcal{C}_{1}$ and $\mathcal{C}_{2}$ defined below. We set $N_{\varepsilon}:=\xi\left(R_{0}^{\varepsilon}\right)$. Then, using independence and that $N_{\varepsilon}$ is a Poisson r.v. with parameter $\lambda \varepsilon^{d}$, we get

$$
\begin{aligned}
\mathcal{C}_{1} & :=\mathbb{E}_{\lambda}\left[\int_{R_{0}^{\varepsilon}} \xi(d x)\left|\operatorname{Piv}_{+}\left(x \leftrightarrow S_{n}(x)\right)\right| \mathbb{1}\left(N_{\varepsilon} \geq 2\right)\right] \\
& \leq \mathbb{E}_{\lambda}\left[\xi\left(\Lambda_{n+2}\right) N_{\varepsilon} \mathbb{1}\left(N_{\varepsilon} \geq 2\right)\right]=\mathbb{E}_{\lambda}\left[\left(N_{\varepsilon}+\xi\left(\Lambda_{n+2} \backslash R_{0}^{\varepsilon}\right)\right) N_{\varepsilon} \mathbb{1}\left(N_{\varepsilon} \geq 2\right)\right] \\
& \leq \mathbb{E}_{\lambda}\left[N_{\varepsilon}^{2} \mathbb{1}\left(N_{\varepsilon} \geq 2\right)\right]+c_{1} \mathbb{E}_{\lambda}\left[N_{\varepsilon} \mathbb{1}\left(N_{\varepsilon} \geq 2\right)\right] \leq c_{2} \mathbb{E}_{\lambda}\left[N_{\varepsilon}^{2} \mathbb{1}\left(N_{\varepsilon} \geq 2\right)\right] \\
& =c_{2}\left(\mathbb{E}_{\lambda}\left[N_{\varepsilon}^{2}\right]-\mathbb{P}_{\lambda}\left[N_{\varepsilon}=1\right]\right)=c_{2}\left(\lambda \varepsilon^{d}+\lambda^{2} \varepsilon^{2 d}-\lambda \varepsilon^{d} e^{-\lambda \varepsilon^{d}}\right)=O\left(\varepsilon^{2 d}\right) .
\end{aligned}
$$

Remark 7.1. For the first inequality in (7.3) we point out that, given $x \in R_{0}^{\varepsilon} \cap \xi$, the set $\operatorname{Piv}_{+}\left(x \leftrightarrow S_{n}(x)\right)$ (referred to $\mathcal{G}$ ) must be contained in $\xi \cap \Lambda_{n+2}$. Indeed, if we take a path in $\mathcal{G}$ from $x$ to the complement of $x+(-n, n)^{d}$ and call $y$ the first vertex of the path outside $x+(-n, n)^{d}$, then the euclidean distance between $y$ and $x+(-n, n)^{d}$ is smaller than 1 (recall that all edges in $\mathcal{G}$ have length smaller than 1). In particular, we have that $\|y\|_{\infty}<\|x\|_{\infty}+n+1 \leq n+2$. As a consequence, 
to know if $x \leftrightarrow S_{n}(x)$ in $\mathcal{G}$ (or in the graph obtained by removing from $\mathcal{G}$ a vertex $z$ and the edges containing $z$ ), it is enough to know the vertexes of $\mathcal{G}$ inside $\Lambda_{n+2}$ and the edges formed by these vertexes.

We now bound the remaining contribution $\mathcal{C}_{2}$ :

$$
\begin{aligned}
\mathcal{C}_{2} & :=\mathbb{E}_{\lambda}\left[\int_{R_{0}^{\varepsilon}} \xi(d x)\left|\operatorname{Piv}_{+}\left(x \leftrightarrow S_{n}(x)\right)\right| \mathbb{1}\left(N_{\varepsilon}=1\right)\right] \\
& =\mathbb{E}_{\lambda}\left[\left|\operatorname{Piv}_{+}\left(\overline{0} \leftrightarrow S_{n}(\overline{0})\right)\right| \mathbb{1}\left(N_{\varepsilon}=1\right)\right]=\mathbb{E}_{\lambda}\left[\left|\operatorname{Piv}_{+}\left(\overline{0} \leftrightarrow S_{n}(\overline{0})\right)\right| \mathbb{1}\left(N_{\varepsilon}=1\right) \mathbb{1}\left(A_{\varepsilon}\right)\right] \\
& +\mathbb{E}_{\lambda}\left[\left|\operatorname{Piv}_{+}\left(\overline{0} \leftrightarrow S_{n}(\overline{0})\right)\right| \mathbb{1}\left(N_{\varepsilon}=1\right) \mathbb{1}\left(A_{\varepsilon}^{c}\right)\right] .
\end{aligned}
$$

We note that (see also the computation of $\mathbb{E}_{\lambda}\left[N_{\varepsilon}^{2} \mathbb{1}\left(N_{\varepsilon} \geq 2\right)\right]$ in (7.3))

$$
\begin{aligned}
& \mathbb{E}_{\lambda}\left[\left|\operatorname{Piv}_{+}\left(\overline{0} \leftrightarrow S_{n}(\overline{0})\right)\right| \mathbb{1}\left(N_{\varepsilon}=1\right) \mathbb{1}\left(A_{\varepsilon}^{c}\right)\right] \leq \mathbb{E}_{\lambda}\left[\xi\left(\Lambda_{n+2}\right) \mathbb{1}\left(N_{\varepsilon}=1\right) \mathbb{1}\left(A_{\varepsilon}^{c}\right)\right] \\
& \quad \leq \sum_{y \in \Gamma_{\varepsilon} \backslash\{0\}} \mathbb{E}_{\lambda}\left[\xi\left(\Lambda_{n+2}\right) \mathbb{1}\left(N_{\varepsilon}=1\right) \mathbb{1}\left(\xi\left(R_{y}^{\varepsilon}\right) \geq 2\right)\right] \\
& \quad \leq \sum_{y \in \Gamma_{\varepsilon} \backslash\{0\}} \mathbb{E}_{\lambda}\left[\xi\left(\Lambda_{n+2} \backslash\left(R_{y}^{\varepsilon} \cup R_{0}^{\varepsilon}\right)\right)\right] \mathbb{P}_{\lambda}\left(N_{\varepsilon}=1\right) \mathbb{P}_{\lambda}\left(\xi\left(R_{y}^{\varepsilon}\right) \geq 2\right) \\
& \quad+2 \sum_{y \in \Gamma_{\varepsilon} \backslash\{0\}} \mathbb{E}_{\lambda}\left[\xi\left(R_{y}^{\varepsilon}\right) \mathbb{1}\left(\xi\left(R_{y}^{\varepsilon}\right) \geq 2\right)\right] \mathbb{P}_{\lambda}\left(N_{\varepsilon}=1\right) \leq \sum_{y \in \Gamma_{\varepsilon} \backslash\{0\}} O\left(\varepsilon^{3 d}\right)=O\left(\varepsilon^{2 d}\right) .
\end{aligned}
$$

Since $(7.2)=\mathcal{C}_{1}+\mathcal{C}_{2}$, by $(7.3),(7.4)$ and $(7.5)$, we get (note that $B_{\varepsilon}=\left\{N_{\varepsilon}=1\right\}$ )

$$
\mathbb{E}_{0, \lambda}\left[\left|\operatorname{Piv}_{+}\left(0 \leftrightarrow S_{n}\right)\right|\right]=\mathbb{E}_{\lambda}\left[\left|\operatorname{Piv}_{+}\left(\overline{0} \leftrightarrow S_{n}(\overline{0})\right)\right| \mathbb{1}\left(A_{\varepsilon} \cap B_{\varepsilon}\right)\right] \cdot \frac{1}{\lambda \varepsilon^{d}}+o(1) .
$$

In what follows, given one of our random graphs on the grid $\Gamma_{\varepsilon}$ as $G_{\varepsilon}$ (cf. Section 3.2), $\mathcal{G}_{\varepsilon}, \mathcal{G}_{\varepsilon}^{*}$ and $\mathcal{G}_{\varepsilon}^{\#}$ (cf. Section 6), and given an event $A$ regarding the graph, we call $\operatorname{Piv}_{+}(A)$ the set of vertexes $x$ of the graph for which the following property holds: the event $A$ is realized by the graph under consideration, but it does

not take place when removing from the graph the vertex $x$ and all edges containing the vertex $x$.

\section{Lemma 7.2. It holds}

$$
\begin{aligned}
\mathbb{E}_{\lambda}\left[\left|\operatorname{Piv}_{+}\left(\overline{0} \leftrightarrow S_{n}(\overline{0})\right)\right| \mathbb{1}\left(A_{\varepsilon} \cap B_{\varepsilon}\right)\right]= & \\
& \mathbb{E}_{\lambda}\left[\mid P i v_{+}\left(0 \leftrightarrow S_{n} \text { in } \mathcal{G}_{\varepsilon}^{*}\right) \mid \mathbb{1}\left(A_{\varepsilon} \cap B_{\varepsilon}\right)\right]+o\left(\varepsilon^{d}\right) .
\end{aligned}
$$

Proof: We can bound

$$
\begin{aligned}
& \mathbb{E}_{\lambda}\left[\left|\operatorname{Piv}_{+}\left(\overline{0} \leftrightarrow S_{n}(\overline{0})\right)\right| \mathbb{1}\left(A_{\varepsilon} \cap B_{\varepsilon}\right) \mathbb{1}\left(\xi\left(\Lambda_{n+\varepsilon} \backslash \stackrel{\circ}{\Lambda}_{n-\varepsilon}\right) \geq 1\right)\right] \\
& \leq \mathbb{E}_{\lambda}\left[\xi\left(\Lambda_{n+2}\right) \mathbb{1}\left(A_{\varepsilon} \cap B_{\varepsilon}\right) \mathbb{1}\left(\xi\left(\Lambda_{n+\varepsilon} \backslash \stackrel{\circ}{\Lambda}_{n-\varepsilon}\right) \geq 1\right)\right] \\
& \leq \mathbb{E}_{\lambda}\left[W \mathbb{1}\left(B_{\varepsilon}\right) \mathbb{1}\left(\xi\left(\Lambda_{n+\varepsilon} \backslash \stackrel{\circ}{\Lambda-\varepsilon}_{n-\varepsilon}\right) \geq 1\right)\right]+\mathbb{E}_{\lambda}\left[\xi\left(\Lambda_{n+\varepsilon} \backslash \stackrel{\circ}{\Lambda}_{n-\varepsilon}\right) \mathbb{1}\left(B_{\varepsilon}\right)\right] \\
& \leq c \mathbb{P}_{\lambda}\left(B_{\varepsilon}\right) \mathbb{P}_{\lambda}\left(\xi\left(\Lambda_{n+\varepsilon} \backslash \stackrel{\circ}{\Lambda}_{n-\varepsilon}\right) \geq 1\right)+\mathbb{E}_{\lambda}\left[\xi\left(\Lambda_{n+\varepsilon} \backslash \stackrel{\circ}{\Lambda}_{n-\varepsilon}\right)\right] \mathbb{P}_{\lambda}\left(B_{\varepsilon}\right) \\
& =O\left(\varepsilon^{d+1}\right)=o\left(\varepsilon^{d}\right) \text {, }
\end{aligned}
$$


where $W:=\xi\left(\Lambda_{n+2} \backslash \Lambda_{n+\varepsilon}\right)+\xi\left(\stackrel{\Lambda}{n-\varepsilon}_{n-\varepsilon} \backslash R_{0}^{\varepsilon}\right)+1$ (note that the third inequality follows from the independence property of the Poisson point process). As a consequence, (7.7) follows by observing that

$$
\begin{aligned}
\mathbb{E}_{\lambda}\left[\left|\operatorname{Piv}_{+}\left(\overline{0} \leftrightarrow S_{n}(\overline{0})\right)\right| \mathbb{1}\left(A_{\varepsilon} \cap B_{\varepsilon}\right) \mathbb{1}\left(\xi\left(\Lambda_{n+\varepsilon} \backslash \stackrel{\circ}{\Lambda}_{n-\varepsilon}\right)=0\right)\right]= \\
\mathbb{E}_{\lambda}\left[\mid \operatorname{Piv}_{+}\left(0 \leftrightarrow S_{n} \text { in } \mathcal{G}_{\varepsilon}^{*}\right) \mid \mathbb{1}\left(A_{\varepsilon} \cap B_{\varepsilon}\right) \mathbb{1}\left(\xi\left(\Lambda_{n+\varepsilon} \backslash \stackrel{\circ}{\Lambda-\varepsilon}_{n-\varepsilon}\right)=0\right)\right] .
\end{aligned}
$$

Let us justify the above observation. We assume that event $A_{\varepsilon} \cap B_{\varepsilon}$ is fulfilled and that $\xi\left(\Lambda_{n+\varepsilon} \backslash \Lambda_{n-\varepsilon}\right)=0$. Recall that $\mathcal{G}_{\varepsilon}^{*}$ is obtained by restricting the graph $\mathcal{G}$ to $\Lambda_{n+1}$ and by sliding any vertex $\bar{x}$ to $x$. Since $S_{n}(\overline{0}) \subset \Lambda_{n+\varepsilon} \backslash \stackrel{\circ}{\Lambda-\varepsilon}_{n-\varepsilon}$, if $\overline{0} \leftrightarrow S_{n}(\overline{0})$ in $\mathcal{G}$ then $\overline{0} \leftrightarrow y$ for some point $y \in \Lambda_{n-\varepsilon+1} \backslash \Lambda_{n+\varepsilon}$ (using that edges have length smaller than 1). It must be $y=\bar{v}$ for some $v \in \Gamma_{\varepsilon}$. Since $\|y-v\|_{\infty} \leq \varepsilon$, we conclude that $v \in \Lambda_{n+1} \backslash \Lambda_{n}$. Since we can restrict to paths from 0 to $y$ with intermediate points lying inside $\Lambda_{n-\varepsilon}$, we have that all the intermediate points are of the form $\bar{z}$ for some $z \in \Gamma_{\varepsilon}$. We therefore get that the above path realizing the event $\overline{0} \leftrightarrow S_{n}(\overline{0})$ in $\mathcal{G}$ corresponds to a path in $\mathcal{G}_{\varepsilon}^{*}$ from 0 to $v,\|v\|_{\infty} \geq n$. On the other hand, since $\xi\left(\Lambda_{n+\varepsilon} \backslash \stackrel{\circ}{\Lambda}_{n-\varepsilon}\right)=0$, any path in $\mathcal{G}_{\varepsilon}^{*}$ from 0 to $v$, with $\|v\|_{\infty} \geq n$, is obtained by sliding some path in $\mathcal{G}$ from $\overline{0}$ to $\Lambda_{n+\varepsilon}^{c}$. As $S_{n}(\overline{0}) \subset \Lambda_{n+\varepsilon} \backslash \stackrel{\circ}{\Lambda-\varepsilon}_{n-\varepsilon}$, these paths in $\mathcal{G}$ must realize the event $\overline{0} \leftrightarrow S_{n}(\overline{0})$. This correspondence between paths implies a correspondence between $(+)$-pivotal points, leading to identity (7.9).

In the last term in (7.7) we can replace $\mathcal{G}_{\varepsilon}^{*}$ with $\mathcal{G}_{\varepsilon}^{\#}$, since they have the same law under $\mathbb{P}_{\lambda}$ conditioned to $\xi$. Now we would like to replace $\mathcal{G}_{\varepsilon}^{\#}$ with $\mathcal{G}_{\varepsilon}$. This is possible due to Lemma 6.4. Indeed, we have

$$
\mathbb{P}_{\lambda}\left(A_{\varepsilon} \cap B_{\varepsilon}\right)=\mathbb{P}_{\lambda}\left(B_{\varepsilon}\right)\left[1-\mathbb{P}_{\lambda}\left(A_{\varepsilon}^{c} \mid B_{\varepsilon}\right)\right]=\mathbb{P}_{\lambda}\left(B_{\varepsilon}\right)(1+o(1))=\lambda \varepsilon^{d}(1+o(1)),
$$

thus implying that Lemma 6.4 is equivalent to the property

$$
\mathbb{E}_{\lambda}\left[\xi\left(\Lambda_{n+2}\right) \mathbb{1}\left(A_{\varepsilon} \cap B_{\varepsilon}\right) \mathbb{1}\left(\mathcal{G}_{\varepsilon} \neq \mathcal{G}_{\varepsilon}^{\#}\right)\right]=o\left(\varepsilon^{d}\right) .
$$

By combining (7.6), (7.7), (7.10) and (7.11) we conclude that

$$
\mathbb{E}_{0, \lambda}\left[\left|\operatorname{Piv}_{+}\left(0 \leftrightarrow S_{n}\right)\right|\right]=\mathbb{E}_{\lambda}\left[\mid \operatorname{Piv}_{+}\left(0 \leftrightarrow S_{n} \text { in } \mathcal{G}_{\varepsilon}\right)|| A_{\varepsilon} \cap B_{\varepsilon}\right]+o(1) .
$$

Due to the definition of the graph $G_{\varepsilon}$ built on $\left(\Omega_{\varepsilon}, \mathbb{P}_{\lambda}^{(\varepsilon)}\right)$ we have

$$
\mathbb{E}_{\lambda}\left[\mid \operatorname{Piv}_{+}\left(0 \leftrightarrow S_{n} \text { in } \mathcal{G}_{\varepsilon}\right)|| A_{\varepsilon} \cap B_{\varepsilon}\right]=\mathbb{E}_{\lambda}^{(\varepsilon)}\left[\left|\operatorname{Piv}_{+}\left(0 \leftrightarrow S_{n}\right)\right| \mid \eta_{0}^{\varepsilon}=1\right]
$$

Above, and in what follows, events appearing in $\mathbb{E}_{\lambda}^{(\varepsilon)}, \mathbb{P}_{\lambda}^{(\varepsilon)}$ are referred to the graph $G_{\varepsilon}$.

By combining (7.12) and (7.13) we have achieved that

$$
\mathbb{E}_{0, \lambda}\left[\left|\operatorname{Piv}_{+}\left(0 \leftrightarrow S_{n}\right)\right|\right]=\lim _{\varepsilon \downarrow 0} \mathbb{E}_{\lambda}^{(\varepsilon)}\left[\left|\operatorname{Piv}_{+}\left(0 \leftrightarrow S_{n}\right)\right| \mid \eta_{0}^{\varepsilon}=1\right] .
$$

To derive (7.1) from (7.14) it is enough to apply the following result: 
Lemma 7.3. It holds $\mathbb{E}_{\lambda}^{(\varepsilon)}\left[\left|\operatorname{Piv}_{+}\left(0 \leftrightarrow S_{n}\right)\right| \mid \eta_{0}^{\varepsilon}=1\right]=\mathbb{E}_{\lambda}^{(\varepsilon)}\left[\left|\operatorname{Piv}\left(0 \leftrightarrow S_{n}\right)\right|\right]$.

Proof: Using the fact that $\left\{\operatorname{Piv}_{+}\left(0 \leftrightarrow S_{n}\right)\right\}$ is empty if $\eta_{0}^{\varepsilon} \neq 1$, we get

$$
\begin{aligned}
\mathbb{E}_{\lambda}^{(\varepsilon)}\left[\left|\operatorname{Piv}_{+}\left(0 \leftrightarrow S_{n}\right)\right| \mid \eta_{0}^{\varepsilon}=1\right] & =\frac{1}{p_{\lambda}(\varepsilon)} \sum_{x \in \Gamma_{\varepsilon}} \mathbb{E}_{\lambda}^{(\varepsilon)}\left[\mathbb{1}\left(x \in \operatorname{Piv}_{+}\left(0 \leftrightarrow S_{n}\right)\right) \eta_{0}^{\varepsilon}\right] \\
& =\frac{1}{p_{\lambda}(\varepsilon)} \sum_{x \in \Gamma_{\varepsilon}} \mathbb{E}_{\lambda}^{(\varepsilon)}\left[\mathbb{1}\left(x \in \operatorname{Piv}_{+}\left(0 \leftrightarrow S_{n}\right)\right)\right] \\
& =\frac{1}{p_{\lambda}(\varepsilon)} \sum_{x \in \Gamma_{\varepsilon}} \mathbb{E}_{\lambda}^{(\varepsilon)}\left[\mathbb{1}\left(x \in \operatorname{Piv}\left(0 \leftrightarrow S_{n}\right)\right) \eta_{x}^{\varepsilon}\right] .
\end{aligned}
$$

Since the events $\left\{x \in \operatorname{Piv}\left(0 \leftrightarrow S_{n}\right)\right\}$ and $\left\{\eta_{x}^{\varepsilon}=1\right\}$ are independent, the last expression equals $\sum_{x \in \Gamma_{\varepsilon}} \mathbb{E}_{\lambda}^{(\varepsilon)}\left[\mathbb{1}\left(x \in \operatorname{Piv}\left(0 \leftrightarrow S_{n}\right)\right)\right]$, thus concluding the proof.

\section{Acknowledgements}

The authors thank H. Duminil-Copin and R. Meester for useful discussions. We also thank the anonymous referee for the careful reading of the manuscript and for his/her corrections and suggestions.

\section{References}

V. Ambegaokar, B. I. Halperin and J. S. Langer. Hopping Conductivity in Disordered Systems. Phys. Rev. B 4, 2612-2620 (1971). DOI: 10.1103/PhysRevB.4.2612.

D. J. Daley and D. Vere-Jones. An introduction to the theory of point processes. Springer Series in Statistics. Springer-Verlag, New York (1988). ISBN 0-38796666-8. MR950166.

H. Duminil-Copin, A. Raoufi and V. Tassion. Exponential decay of connection probabilities for subcritical Voronoi percolation in $\mathbb{R}^{d}$. Probab. Theory Related Fields 173 (1-2), 479-490 (2019a). MR3916112.

H. Duminil-Copin, A. Raoufi and V. Tassion. Sharp phase transition for the random-cluster and Potts models via decision trees. Ann. of Math. (2) 189 (1), 75-99 (2019b). MR3898174.

A. Faggionato. Mott's law for the effective conductance of the Miller-Abrahams random resistor network. ArXiv Mathematics e-prints (2017). In preparation. A preliminary version is available online as arXiv: 1712.07980 .

A. Faggionato and P. Mathieu. Mott law as upper bound for a random walk in a random environment. Comm. Math. Phys. 281 (1), 263-286 (2008). MR2403611.

A. Faggionato, H. Schulz-Baldes and D. Spehner. Mott law as lower bound for a random walk in a random environment. Comm. Math. Phys. 263 (1), 21-64 (2006). MR2207323.

J. Jiang, S. Zhang and T. Guo. Russo's formula, uniqueness of the infinite cluster, and continuous differentiability of free energy for continuum percolation. J. Appl. Probab. 48 (3), 597-610 (2011). MR2884802.

R. Meester and R. Roy. Continuum percolation, volume 119 of Cambridge Tracts in Mathematics. Cambridge University Press, Cambridge (1996). ISBN 0-52147504-X. MR1409145. 
A. Miller and E. Abrahams. Impurity Conduction at Low Concentrations. Phys. Rev. 120, 745-755 (1960). DOI: 10.1103/PhysRev.120.745.

R. O'Donnell, M. Saks, O. Schramm and R. Servedio. Every decision tree has an influential variable. In 46th Annual IEEE Symposium on Foundations of Computer Science (FOCS'05), pages 31-39 (2005). DOI: 10.1109/SFCS.2005.34.

M. Pollak, M. Ortuño and A. Frydman. The Electron Glass. Cambridge University Press, United Kingdom, first edition (2013). ISBN 0521195527, 978-0521195522.

S. Shklovskii and A. L. Efros. Electronic Properties of Doped Semiconductors, volume 45 of Springer Series in Solid-State Sciences. Springer Verlag, Berlin, first edition (1984). DOI: 10.1007/978-3-662-02403-4.

S. Ziesche. Sharpness of the phase transition and lower bounds for the critical intensity in continuum percolation on $\mathbb{R}^{d}$. Ann. Inst. Henri Poincaré Probab. Stat. 54 (2), 866-878 (2018). MR3795069. 